\title{
Low Energy Bands do not Contribute to Quantum Hall Effect
}

\author{
S. Nakamura ${ }^{\star}$ and J. Bellissard ${ }^{\star \star}$ \\ Centre de Physique Théorique, ${ }^{\star \star \star}$ CNRS-Luminy, Case 907, F-13288, Marseille, Cedex 09, France
}

\begin{abstract}
Using non-commutative geometry and localization techniques we prove rigorously a result of Tesanovic, Axel and Halperin, namely that low energy bands in an ordered or slightly disordered 2D crystal submitted to a uniform magnetic field, do not contribute to the Hall conductivity.
\end{abstract}

\section{Introduction}

We first consider an electron gas in a perfect two dimensional crystal submitted to a perpendicular uniform magnetic field. If we neglect the Coulomb interaction between pairs of electrons and the motion of ions in the crystal, we are led to study the quantum motion of one charged particle (an electron or a hole) of mass $m$, of charge $\pm e$, in a periodic potential $\mu V(\mathbf{x})$ (here $\mu$ is a coupling constant) and a uniform magnetic field $B$.

For $\mu=0$, the energy spectrum is given by the Landau levels $E_{n}=\hbar \omega_{c}(n+1 / 2)$ where $\omega_{c}$ is the cyclotron frequency $e B / m$ and $n=0,1,2, \ldots$ [1]. The gap between $E_{n-1}$ and $E_{n}$ will be called $G_{n}$. It is well known that whenever the Fermi level is in $G_{n}$ the quantum Hall conductance at zero temperature is quantized and equal to $n e^{2} / h$ ( $h$ is the Planck constant). Both $G_{n}$ and that quantization do survive for $\mu>0$ small enough. This fact has been explained by recognizing that up to the universal physical constant $e^{2} / h$ the Hall conductance at zero temperature is equal to the Chern class of the eigenprojection $P_{F}$ of the Hamiltonian up to energies smaller than or equal to the Fermi level [2-8].

In a recent paper, B. Halperin et al. [9] have investigated through a numerical calculation how that scheme is modified as $\mu$ increases from zero to infinity for a genuine periodic potential $V$. For $\mu$ small, Landau levels broaden into bands $B_{n}$ of width of order $\mu$. Their structure is actually quite complicated for it is given in

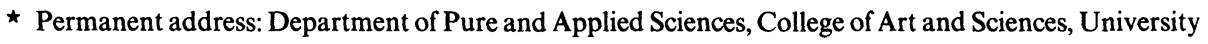
of Tokyo, Komaba, 3-8-1, Meguro-Ku, Tokyo 153, Japan

$\star \star$ Université de Provence, Marseille

$\star \star \star$ Laboratoire Propre, Centre National de la Recherche Scientifique 
first approximation by the spectrum of a Harper-like equation [7, 10-12] and is believed to be a Cantor set of zero Lebesgue measure at least if the crystal is isotropic $[13,14]$. This has actually been proved rigorously for a generic set of values of the magnetic field and in a certain number of examples $[12,15-18]$.

If $\mu$ increases, Halperin et al. found that eventually the bands $B_{n-1}$ and $B_{n}$ cross each other for some value $\mu_{n}^{-}$of $\mu$. On the other hand for $\mu>\mu_{n}^{+}>\mu_{n}^{-}$these bands separate again in a way which may become complicated for high $n$ 's. The quantum Hall conductance is found to vanish if the Fermi level belongs to $G_{n}$ for $\mu>\mu_{n}^{+}$. This is a clear indication that the crossings observed are not artefacts of the numerical calculation because of the conservation laws valid for the quantum Hall conductance $[3,4,6,7]$.

Our aim in this paper is to prove this result on a mathematically rigorous ground. We will actually show that it still holds even if the crystal is not perfectly periodic. Our only requirement is that the atoms be identical and separated by a distance uniformly bounded from below with probability one. Then we prove that for any energy $E_{c}>0$ there is a coupling constant $\mu_{c}$ beyond which in the energy range $\left(-\infty, E_{c} \mu^{1 / 2}\right]$ the energy spectrum splits into well separated bands $B_{n, n^{\prime}}$. They are located around the energies $E_{n, n^{\prime}}$ and have a width $\Delta_{n, n^{\prime}}$ given by

$$
\begin{aligned}
& E_{n, n^{\prime}}=\mu^{1 / 2} \hbar\left\{(n+1 / 2) \omega_{1}+\left(n^{\prime}+1 / 2\right) \omega_{2}\right\}+e_{n, n^{\prime}}+O\left(\mu^{-1 / 2}\right), \\
& \Delta_{n, n^{\prime}}=O\left\{\exp \left(- \text { const. } \mu^{1 / 2}\right)\right\} \quad n, n^{\prime} \in \mathbf{N},
\end{aligned}
$$

where $\omega_{1}, \omega_{2}$ are the eigenvalues of the Hessian $\partial^{2} V / \partial \mathbf{x} \partial \mathbf{x}$ of the potential at its minimum, and $e_{n, n^{\prime}}$ is the contribution of the anharmonic perturbation at the bottom well. If the potential is rotation invariant the energy bands look like

$$
E_{n, k}=\mu^{1 / 2} \hbar(n+1 / 2) \omega+e_{n, k}+O\left(\mu^{-1 / 2}\right)
$$

In addition, we show that the eigenprojectors on each of these bands have zero Chern class. In other words, the low lying energy bands at high coupling do not contribute to the quantum Hall conductance.

The main reason for these results come from the remark that the limit $\mu \rightarrow \infty$ is nothing but the semiclassical limit with a Planck constant proportional to $\mu^{-1 / 2}$. In that limit all potential wells get deeper and deeper as if the atoms were separated from each other. The lowest energy levels are then given by the harmonic approximation in two dimensions at lowest order in $\mu^{-1 / 2}$. If the atomic potential is rotation invariant, each level has a degeneracy equal to $n+1$ which may be partially or totally broken at the next order due to anharmonic terms in the potential. The band width is due to tunnelling between the wells and is therefore at most of exponential order in $\mu^{1 / 2}$.

The vanishing of the Chern class is obtained by remarking that as $\mu \rightarrow \infty$ the eigenprojections on each of the previous bands do converge in norm to the atomic eigenprojection for the crystal obtained with perfectly isolated atoms. This latter projection is the direct sum of atomic projections having a vanishing Chern class which expresses that the current cannot go through if the atoms are perfectly isolated. By homotopy invariance, we conclude that the same is true at finite $\mu$ 's as long as the bands are not crossing.

We emphasize that the vanishing of the quantum Hall conductance need not 
imply the occurrence of localized states. Actually if the potential is periodic, using a Landau gauge, the Hamiltonian becomes translation invariant in one direction giving rise to states extended in that direction. Localization, when it occurs, is mainly due to disorder which prevent Bragg's condition to hold for wave functions.

Technically we have chosen to use the semiclassical framework developped by Briet-Combes-Duclos [19] rather than the pseudodifferential operator formalism of Helffer-Sjöstrand [12, 20]. This is because the former approach is closer to the operator algebra techniques previously used in proving the existence and the quantization of plateaus for the quantum Hall conductance $[5,6]$. It consists in separating the wells by mean of a non-commutative partition of the unity and comparing the total Hamiltonian of the system with the direct sum of individual atomic Hamiltonians. To this end we use an algebraic endomorphism of the original observable algebra into the algebra of the isolated atoms. It has the advantage of not changing the formulæ involved in proving the integrality of the Chern number. It is actually remarkable that this formalism fits so well with the algebraic framework.

The paper is organized as follows. In Sect. 2, we describe the model and our results precisely. Section 3 is devoted to the description of an observable algebra in order to define the conductance properly. In Sect. 4 we construct an individual atomic system approximating the original one, and some related operator algebras are introduced in Sect. 5. The vanishing of the Chern class projection is proved in Sect. 6, and Sect. 7 gives the technical estimates required in Sect. 6. Several appendices gives proofs of some well-known results which have not been actually published.

\section{Model and Main Results}

We consider a two-dimensional Schrödinger operator in a uniform magnetic field:

$$
H=-(\partial / \partial \mathbf{x}-\mathbf{i A}(\mathbf{x}))^{2}+\mu V(\mathbf{x})
$$

acting on $\mathscr{H}=L^{2}\left(\mathbf{R}^{2}\right)$, where the units are chosen such that mass of the particle is $1 / 2$, its electric charge is 1 and the Planck constant is 1 . In (2.1), $\partial / \partial \mathbf{x}=\left(\partial / \partial x_{1}, \partial / \partial x_{2}\right)$ is the gradient operator and $\mathbf{A}(\mathbf{x})=\left(-B x_{2} / 2, B x_{1} / 2\right)$ is the vector potential (thus $\partial_{1} A_{2}-\partial_{2} A_{1}=B$ ). It is well-known that $H$ is essentially self-adjoint on $\mathscr{C}_{0}^{\infty}\left(\mathbf{R}^{2}\right)$ [21].

We consider the potential $V=V_{\omega}$ as a random process on a probability space $\Omega$. $\Omega$ can be considered as the set of impurity configurations, hence it is natural to introduce an action of the translation group $\left\{T^{a} ; \mathbf{a} \in \mathbf{R}^{2}\right\}$ on $\Omega$. We suppose that this action leaves the probability measure $\mathbf{P}$ on $\Omega$ invariant, and acts ergodically. We introduce the so-called "magnetic translation" operator [22]:

$$
\{U(\mathbf{a}) \psi\}(\mathbf{x})=e^{i B \mathbf{x} \wedge \mathbf{a} / 2} \psi(\mathbf{x}-\mathbf{a}), \quad \psi \in \mathscr{H},
$$

where $\mathbf{x} \wedge \mathbf{y}=x_{1} y_{2}-x_{2} y_{1}$, then the action $T^{\mathbf{a}}$ should satisfy the following homogeneity condition:

$$
U(\mathbf{a}) H_{\omega} U(\mathbf{a})^{*}=H_{T^{\mathbf{a}} \omega}, \quad \omega \in \Omega, \quad \mathbf{a} \in \mathbf{R}^{2},
$$


where $H_{\omega}$ is the Hamiltonian with $V=V_{\omega}$ corresponding to $\omega \in \Omega$. We may consider (2.3) as an assumption the action $\left\{T^{\mathbf{a}}\right\}$ should satisfy. Note that $\left\{U(\mathbf{a}) ; \mathbf{a} \in \mathbf{R}^{2}\right\}$ form a non-commutative group [22]. Further we assume that $V=V_{\omega}$ satisfies almost surely the following assumptions:

\section{Assumption A}

(i) $\inf V(\mathbf{x})=0, \sup _{x \in \mathbf{R}^{2}} V(\mathbf{x}) \leqq C<\infty$.

(ii) There is a countable set of $\mathbf{R}^{2},\left\{\mathbf{x}_{n} ; n=1,2, \ldots\right\}$ such that $\left|\mathbf{x}_{n}-\mathbf{x}_{m}\right| \geqq d$ if $n \neq m$ with $d>0$.

(iii) There are $\varepsilon>0$ and $\mathscr{V} \in \mathscr{C}^{2}(\{\mathbf{x}|| \mathbf{x} \mid<\varepsilon\})$ such that $d>2 \varepsilon$ and $V\left(\mathbf{x}_{n}+\mathbf{x}\right)=\mathscr{V}(\mathbf{x})$ if $|\mathbf{x}|<\varepsilon$ for all $n$.

(iv) 0 is the unique nondegenerate minimum of $\mathscr{V}$ in $\{\mathbf{x}|| \mathbf{x} \mid<\varepsilon\}$.

(v) If $\left|\mathbf{x}_{n}-\mathbf{x}\right|>\varepsilon$ for all $n$ 's then $V(\mathbf{x}) \geqq \delta>0$.

(vi) The constants $C, d, \varepsilon$ and $\delta$ are independent of $\omega \in \Omega$ almost surely.

In the previous framework one can actually identify $\Omega$ with the set of potential $V_{\omega}$, namely a set of bounded multiplication operators on $\mathscr{H}$ (by Assumption A-(i)). If we endow it with the weak topology its closure is a compact space. By ergodicity of the translation group, we can actually identify $\Omega$ with the weak closure of the set $\left\{V_{\omega}(.-\mathbf{a}) ; \mathbf{a} \in \mathbf{R}^{2}\right\}$ for one configuration $\omega$ of the impurity. Actually the boundedness of $V$ is not essential provided it is relatively bounded with respect to the free Laplacian.

Example. Let us consider a periodic potential $V$ with lattice of periods (Bravais lattice) $\Gamma$. Then we can take $\Omega=\mathbf{R}^{2} / \Gamma$ and $T^{\mathbf{a}}$ is the natural action of $\mathbf{R}^{2}$ on $\Omega$. It is easy to check that (2.3) holds in this case. A typical example is:

$$
V(\mathbf{x})=\sin x_{1}+\sin x_{2}+2, \quad \text { or } \quad V_{\omega}=\sin \left(x_{1}+\omega_{1}\right)+\sin \left(x_{2}+\omega_{2}\right)+2
$$

with $\mathbf{x} \in \mathbf{R}^{2}, \omega \in \Omega=\mathbf{R}^{2} / 2 \pi \mathbf{Z}^{2}$, and this is what Halperin et al. studied in [9].

Let $F$ be the Fermi energy level, and let $P_{F}$ be the eigenprojection of the Hamiltonian on the subset $(-\infty, F]$ of energies, namely $P_{F}=E_{(-\infty, F)}(H)$. A "differential" $\partial_{i}(i=1,2)$ is defined by:

$$
\partial_{i} A=2 \pi \mathrm{i}\left[X_{i}, A\right], \quad i=1,2
$$

for an operator $A$, where $\mathbf{X}=\left(X_{1}, X_{2}\right)$ is the position operator and $[A, B]=A B-B A$ denotes the commutator (this is defined as a form on the domain $D(A) \cap D(|\mathbf{X}|)$ at first and then extended to an operator. We will see later that this is possible for a class of operators including $P_{F}$, if $F$ is in a gap of the spectrum). The "trace per unit area" is defined by:

$$
\tau(A)=\lim _{|\Lambda| \rightarrow \infty}|\Lambda|^{-1} \operatorname{Tr}_{\Lambda}(A),
$$

where $\Lambda$ is a square with center at the origin, and $\operatorname{Tr}_{\Lambda}$ is the usual trace in $L^{2}(\Lambda)$. Note that if $A=P_{F}, \tau\left(P_{F}\right)$ is the integrated density of states at the energy $F$. Again we will see later that $\tau(A)$ exist for a class of operators, and it is independent upon the choice of $\omega \in \Omega$ if $A=A(\omega)$ satisfies the homogeneity condition (2.3) (see Sect. 3). 
Now, the Hall conductivity $\sigma_{H}$ at zero temperature is defined by:

$$
\sigma_{H}=1 / 2 \mathbf{i} \pi \tau\left(P_{F}\left[\partial_{1} P_{F}, \partial_{2} P_{F}\right]\right)
$$

by virtue of Kubo's formula [23, 2, 4, 6, 8].

We defined the one-ion Hamiltonian as:

$$
h=-(\partial / \partial \mathbf{x}-\mathbf{i A}(\mathbf{x}))^{2}+\mu \mathscr{V}(\mathbf{x})
$$

on $L^{2}\left(B_{\varepsilon}\right), B_{\varepsilon}=\{\mathbf{x} ;|\mathbf{x}|<\varepsilon\}$ with the Dirichlet boundary conditions. It is well-known that $h$ has a discrete spectrum. Moreover, if $E_{k}(\mu)$ is the $k^{\text {th }}$ eigenvalue of $h$, it admits an asymptotic expansion

$$
E_{k}(\mu) \approx \sum_{i=0}^{\infty} C_{i} \mu^{-(2+i) / 4}, \quad \mu->\infty
$$

by the harmonic approximation at $\mathbf{x}=0[24,20]$. In particular, the top term $C_{0}$ is the $k^{\text {th }}$ lowest number in $\left\{(i+1 / 2) \omega_{1}+(j+1 / 2) \omega_{2} ; i, j=0,1,2, \ldots\right\}$, where $\omega_{1}, \omega_{2}$ are the eigenvalues of the Hessian $\partial^{2} \mathscr{V} / \partial \mathbf{x} \partial \mathbf{x}$ of the potential at $\mathbf{x}=0$.

Our main result is:

Theorem 1. There exist $a>0$ such that if a Fermi Energy $F$ satisfies $F \leqq o(\mu)$ and if

$$
\operatorname{dist}(F, \sigma(h)) \geqq \exp \left(-a \mu^{1 / 2}\right),
$$

then $F$ lies in a gap of the spectrum of $H$ almost surely, and the Hall conductivity vanishes i.e. $\sigma_{H}=0$, for sufficiently large $\mu$.

The former statement implies that the width of bands are at most of order $\exp \left(-a \mu^{1 / 2}\right)$ if they correspond to simple eigenvalues of $h$, and the latter that the contribution of that band to the Hall conductivity is zero for large enough $\mu$ 's.

\section{Observable Algebra}

In this section, we consider an algebra, called observable algebra, of operatorvalued functions on $\Omega$ satisfying the homogeneity condition:

$$
U(\mathbf{a}) A(\omega) U(\mathbf{a})^{*}=A\left(T^{\mathbf{a}} \omega\right), \quad \omega \in \Omega, \mathbf{a} \in \mathbf{R}^{2},
$$

where $A: \Omega \rightarrow \mathscr{B}(\mathscr{H})$, following $[5,6]$.

Ar first, let $\mathcal{O}_{0}$ be the set of functions on $\Omega \times \mathbf{R}^{2}$ continuous with compact support. $\mathcal{O}_{0}$ forms a $*$-Algebra with the following operations:

$$
\begin{aligned}
(A B)(\omega, \mathbf{x}) & =\int_{\mathbf{R}^{2}} d^{2} \mathbf{y} A(\omega, \mathbf{x}) B\left(T^{-\mathbf{y}} \omega, \mathbf{x}-\mathbf{y}\right) e^{-i B x \wedge y / 2}, \\
A^{*}(\omega, \mathbf{x}) & =A\left(T^{-\mathbf{x}} \omega,-\mathbf{x}\right)^{*}
\end{aligned}
$$

for $A, B \in \mathcal{O}_{0}$. An element of $\mathcal{O}_{0}$ is represented as an operator-valued function on $\Omega$ by:

$$
\pi_{\omega}(A) \psi(\mathbf{x})=\int_{\mathbf{R}^{2}} d^{2} \mathbf{y} A\left(T^{-\mathbf{x}} \omega, \mathbf{y}-\mathbf{x}\right) e^{i B x \wedge y / 2} \psi(\mathbf{y})
$$

with $\psi \in \mathscr{H}$. It is easy to see that $\pi_{\omega}$ is a $*$-representation of $\mathcal{O}_{0}$ for each $\omega \in \Omega$, i.e. 
(i) it is linear; (ii) $\pi_{\omega}(A) \pi_{\omega}(B)=\pi_{\omega}(A B)$; (iii) $\pi_{\omega}(A)^{*}=\pi_{\omega}\left(A^{*}\right)$. It is also easy to see that $\pi_{\omega}(A)$ satisfies the homogeneity condition (3.1).

Remark 3.1. The mapping $\omega \in \Omega \rightarrow \pi_{\omega}(A)$ is strongly continuous by Lebesgue's dominated convergence theorem.

Proposition 3.1. Let $A=A(\omega)$ be an operator-valued function on $\Omega$, and suppose:

(i) $A$ has an integral kernel $K(\omega ; \mathbf{x}, \mathbf{y})$;

(ii) $K$ is continuous and $K(\omega ; \mathbf{x}, \mathbf{y})=0$ if $|\mathbf{x}-\mathbf{y}| \geqq C$ with $C>0$;

(iii) $A$ satisfies (3.1).

Then $A$ is represented by an element of $\mathcal{O}_{0}$, i.e. $A(\omega)=\pi_{\omega}\left(A^{\sim}\right)$ for some $A^{\sim}$ in $\mathcal{O}_{0}$. Moreover $A^{\sim}(\omega, \mathbf{x})=K(\omega ; 0, \mathbf{x})$.

Proof. By the homogeneity condition, $K$ must satisfy:

$$
K\left(T^{\mathbf{x}} \omega ; \mathbf{x}, \mathbf{x}+\mathbf{y}\right)=K(\omega ; 0, \mathbf{y}) e^{i B \mathbf{x} \wedge \mathbf{y} / 2}
$$

Hence we obtain:

$$
K(\omega ; \mathbf{x}, \mathbf{y})=K\left(T^{-\mathbf{x}} \omega ; 0, \mathbf{y}-\mathbf{x}\right) e^{i B \mathbf{x} \wedge \mathbf{y} / 2}=A^{\sim}\left(T^{-\mathbf{x}} \omega ; 0, \mathbf{y}-\mathbf{x}\right) e^{i B \mathbf{x} \wedge \mathbf{y} / 2}
$$

Comparing this with (3.3) we conclude the proposition.

A $C^{*}$-norm $\|\cdot\|$ on $\mathcal{O}_{0}$ is defined by:

$$
\|A\|=\sup _{\omega \in \Omega}\left\|\pi_{\omega}(A)\right\|_{\mathscr{B}(\mathscr{H})},
$$

and $\mathcal{O}$ is the $C^{*}$-Algebra obtained by completion of $\mathcal{O}_{0}$ with respect to this norm. A trace $\tau$ on $\mathcal{O}_{0}$ is defined by:

$$
\tau(A)=\int_{\omega \in \Omega} \mathbf{P}(d \omega) A(\omega, 0) .
$$

By Birkhoff's mean ergodic theorem $[25,5]$ and the ergodicity of the action $\left\{T^{\mathbf{a}}\right\}$ we get:

$$
\tau(A)=\lim _{|\boldsymbol{\Lambda}| \rightarrow \infty}|\Lambda|^{-1} \int_{\mathbf{a} \in \boldsymbol{\Lambda}} d^{2} \mathbf{a} A\left(T^{\mathbf{a}} \omega, 0\right)=\lim _{|\Lambda| \rightarrow \infty}|\Lambda|^{-1} \operatorname{Tr}_{\Lambda}\left(\pi_{\omega}(A)\right)
$$

for almost all $\omega \in \Omega$, where $\Lambda$ is a cube with center at the origin. It is not too hard to see that $\tau$ is extended to a trace on the $C^{*}$-Algebra $\mathcal{O}$ [26]. We note that $\tau$ given above is identified with $\tau$ defined by (2.6) up to the representation $\pi_{\omega}$ by virtue of the identity (3.8). Hence if the trace norm:

$$
\|A\|_{L^{1}}=\tau\left(\left|A^{*} A\right|^{1 / 2}\right)
$$

is finite, then $\tau(A)$ is well-defined and finite: $|\tau(A)| \leqq\|A\|_{\mathscr{L}}$.

A differential structure is defined by:

$$
\partial_{i} A(\omega, \mathbf{x})=2 \pi \mathbf{i} x_{i} A(\omega, \mathbf{x}), \quad i=1,2 .
$$

By easy calculation, we see that:

$$
\pi_{\omega}\left(\partial_{i} A\right)=\partial_{i} \pi_{\omega}(A)
$$

where $\partial_{i}$ on the right-hand side is defined by (2.5). 
To define the conductivity $\sigma_{H}$, we introduce the "Sobolev space" with respect to the above differential structure. We set:

$$
\|A\|_{1}^{2}=\tau\left(\left|\partial_{1} A\right|^{2}+\left|\partial_{2} A\right|^{2}\right)=4 \pi^{2} \int_{\mathbf{x} \in \mathbf{R}^{2}} d^{2} \mathbf{x} \int_{\omega \in \Omega} \mathbf{P}(d \omega)|\mathbf{x}|^{2}|A(\omega, \mathbf{x})|^{2},
$$

for $A \in \mathcal{O}_{0}$, and $\mathscr{H}^{1}$ is the completion of $\mathcal{O}_{0}$ with respect to this norm in $\mathcal{O}$. Following Connes [27] we introduce the "2-cocycle" $\tau_{2}$ by:

$$
\tau_{2}\left(A_{0}, A_{1}, A_{2}\right)=(1 / 2 \mathbf{i} \pi) \tau\left(A_{0}\left\{\partial_{1} A_{1} \partial_{2} A_{2}-\partial_{2} A_{1} \partial_{1} A_{2}\right\}\right),
$$

for $A \in \mathcal{O}$ and $A_{1}, A_{2} \in \mathscr{H}^{1}$.

Lemma 3.2. $\tau_{2}$ is a bounded form on $\mathcal{O} \times \mathscr{H}^{1} \times \mathscr{H}^{1}$ namely we have:

$$
\left|\tau_{2}\left(A_{0}, A_{1}, A_{2}\right)\right| \leqq(1 / \pi)\left\|A_{0}\right\|\left\|A_{1}\right\|_{1}\left\|A_{2}\right\|_{1} . \diamond
$$

Proof. By properties of trace [26, Chap. 5],

$$
\begin{aligned}
\left|\tau\left(A_{0} \partial_{1} A_{1} \partial_{2} A_{2}\right)\right| & =\left|\tau\left(\left\{\partial_{1} A_{1}^{*} A_{0}^{*}\right\}^{*} \partial_{2} A_{2}\right)\right| \leqq\left|\tau\left(\left|\partial_{1} A_{1}^{*} A_{0}^{*}\right|^{2}\right)\right|^{1 / 2}\left|\tau\left(\left|\partial_{2} A_{2}\right|^{2}\right)\right|^{1 / 2} \\
& =\left|\tau\left(\partial_{1} A_{1}^{*}\left|A_{0}\right|^{2} \partial_{1} A_{1}\right)\right|^{1 / 2}\left|\tau\left(\left|\partial_{2} A_{2}\right|^{2}\right)\right|^{1 / 2} \\
& \leqq\left\|A_{0}\right\|\left|\tau\left(\left|\partial_{1} A_{1}\right|^{2}\right)\right|^{1 / 2}\left|\tau\left(\left|\partial_{2} A_{2}\right|^{2}\right)\right|^{1 / 2} \\
& \leqq\left\|A_{0}\right\|\left\|A_{1}\right\|_{1}\left\|A_{2}\right\|_{1} .
\end{aligned}
$$

Similarly we have:

$$
\left|\tau\left(A_{0} \partial_{2} A_{1} \partial_{1} A_{2}\right)\right| \leqq\left\|A_{0}\right\|\left\|A_{1}\right\|_{1}\left\|A_{2}\right\|_{1} .
$$

Now we will show that $P_{F}$ is represented by an element of $\mathscr{H}^{1}$ whenever $F$ belongs to a gap of the spectrum.

Proposition 3.3. Let $A$ be an operator-valued function on $\Omega$ and suppose:

(i) $A$ has a continuous integral kernel $K(\omega ; \mathbf{x}, \mathbf{y})$; (ii) there is an $L^{1}\left(\mathbf{R}^{2}\right)$-function $f$ such that $|K(\omega ; \mathbf{x}, \mathbf{y})| \leqq f(\mathbf{x}-\mathbf{y})$ for a.e. $\omega, \mathbf{x}, \mathbf{y}$; (iii) $A$ satisfies the homogeneity condition (3.1). Then $A$ is represented by an element of $\mathcal{O}$.

Proof. As in the proof of Proposition 3.1, we can set:

$$
A^{\sim}(\omega, \mathbf{x})=K(\omega ; 0, \mathbf{x}),
$$

as a symbol corresponding to $A$. Clearly $A^{\sim}$ is continuous and $A^{\sim}(\omega) \phi=\pi_{\omega}\left(A^{\sim}\right) \phi$ (see Eq. 3.3) for $\phi \in \mathscr{C}_{0}^{\infty}$. If we set:

$$
A_{\varepsilon}^{\sim}(\omega, \mathbf{x})=\chi(\varepsilon \mathbf{x}) A^{\sim}(\omega, \mathbf{x}),
$$

where $\chi \in C_{0}^{\infty}\left(\mathbf{R}^{2}\right), 0 \leqq \chi \leqq 1$ and $\chi=1$ near 0 , then $A_{\varepsilon}^{\sim} \in \mathcal{O}_{0}$. Since:

we have

$$
\left|A_{\varepsilon}^{\sim}(\omega, \mathbf{x})-A^{\sim}(\omega, \mathbf{x})\right| \leqq(1-\chi(\varepsilon \mathbf{x})) f(\mathbf{x}),
$$

$$
\left\|A^{\sim}-A_{\varepsilon}^{\sim}\right\| \leqq\|(1-\chi(\varepsilon \mathbf{x})) f(\mathbf{x})\|_{\mathscr{L}, 1} \rightarrow 0 \quad \text { as } \quad \varepsilon \rightarrow 0,
$$

and this proves $A^{\sim} \in \mathcal{O}$.

Proposition 3.4. For $z \in \bigcap_{\omega \in \Omega} \rho\left(H_{\omega}\right),\left(H_{\omega}-z\right)^{-1}$ is represented by an element of $\mathscr{H}^{1}$. 
Proof. We set $H_{B}=-(\partial / \partial \mathbf{x}-\mathbf{i A}(\mathbf{x}))^{2}$, and

$$
R_{\varepsilon}(z)=\left(1+\varepsilon H_{B}\right)^{-1}\left(H_{\omega}-z\right)^{-1}\left(1+\varepsilon H_{B}\right)^{-1} .
$$

By Proposition B.1 and Lemmas B.7 in Appendix B, $R_{\varepsilon}(z)$ satisfies the assumptions of Proposition 3.3, hence $R_{\varepsilon}(z) \in \mathcal{O}$ (Proposition B.2). It is easy to see by functional calculus that $R_{\varepsilon}(z)$ converges to $\left(H_{\omega}-z\right)^{-1}$ in norm as $\varepsilon \rightarrow 0$, hence $\left(H_{\omega}-z\right)^{-1} \in \mathcal{O}$ (Proposition B.2). Let $r_{\varepsilon}(\omega, \mathbf{x})$ be the symbol of the element of $\mathcal{O}$ corresponding to $R_{\varepsilon}(z)$. We have by Lemma B.7:

$$
\| r_{\varepsilon}-\left(1-\chi(\delta \mathbf{x}) r_{\varepsilon} \|_{1}^{2} \leqq C \int_{\mathbf{x} \in \mathbf{R}^{2}} e^{-\tau|\mathbf{x}|}(1-\chi(\delta \mathbf{x}))^{2} d^{2} \mathbf{x} \rightarrow 0 \quad \text { as } \quad \delta \rightarrow 0\right.
$$

where $\chi \in \mathscr{C}_{0}^{\infty}$ is that in the proof of Proposition 3.3. This implies $r_{\varepsilon} \in \mathscr{H}^{1}$. Since $r_{\varepsilon}(\omega, \mathbf{x}) \rightarrow r_{0}(\omega, \mathbf{x})$ pointwise if $\mathbf{x} \neq 0$, we can apply the dominated convergence theorem to show:

$$
\left\|r_{\varepsilon}-r_{0}\right\|_{1} \rightarrow 0 \quad \text { as } \quad \varepsilon \rightarrow 0
$$

combining with Lemmas A.8 and B.7. Thus $\left(H_{\omega}-z\right)^{-1}$ is included in the image of $\pi_{\omega}\left(\mathscr{H}^{1}\right)$.

Corollary 3.5. If $F$ belongs to a gap of $\bigcup_{\omega \in \Omega} \sigma\left(H_{\omega}\right)$, then $P_{F}=E_{(-\infty, F)}(H)$ is
represented by an element of $\mathscr{H}^{1} . \diamond$ Combining Lemma 3.2 with Corollary 3.5, we know that the Hall conductivity $\sigma_{H}=\tau_{2}\left(P_{F}, P_{F}, P_{F}\right)$ is well-defined whenever $F$ belongs to a gap of the spectrum.

Remark 3.2. Because of the homogeneity condition (3.1) and the ergodicity of the action $\left\{T^{\mathbf{a}}\right\}$ the spectrum of $H_{\omega}$ is almost surely independent of $\omega \in \Omega$ [28].

\section{Approximation by Individual Atomic Systems}

In this section we fix $V(\mathbf{x})=V_{\omega}(\mathbf{x}), \omega \in \Omega$, and we suppose that Assumption $(A)$ holds for $V(\mathbf{x})$. Let $B_{r}(\mathbf{x})$ be the ball of radius $r$ centered at $\mathbf{x}$, i.e. $B_{r}(\mathbf{x})=\left\{\mathbf{y} \in \mathbf{R}^{2} ;|\mathbf{y}-\mathbf{x}|<r\right\}$, and let $j \in \mathscr{C}_{0}^{\infty}\left(B_{\varepsilon}(0)\right)$ be a cut-off function such that $0 \leqq j(\mathbf{x}) \leqq 1, j(\mathbf{x})=1$ if $|\mathbf{x}| \leqq \varepsilon^{\prime}$ and there is $j^{\prime} \in \mathscr{C}^{\infty}\left(\mathbf{R}^{2}\right)$ such that $j(\mathbf{x})^{2}+j^{\prime}(\mathbf{x})^{2}=1$ for $x \in \mathbf{R}^{2}$, where $\varepsilon^{\prime}$ is an arbitrary fixed constant such that $0<\varepsilon^{\prime}<\varepsilon$. $j_{n}$ and $j_{n}^{\prime}$ are defined by $j_{n}(\mathbf{x})=j\left(\mathbf{x}-\mathbf{x}_{n}\right)$ and $j_{n}^{\prime}(\mathbf{x})=j^{\prime}\left(\mathbf{x}-\mathbf{x}_{n}\right)$ respectively. We also set $j_{0}(\mathbf{x})=\prod_{n=1}^{\infty} j_{n}^{\prime}(\mathbf{x})$ and the gauge transform $g_{n}(\mathbf{x})=\exp \left(\mathbf{i} B \mathbf{x} \wedge \mathbf{x}_{n} / 2\right)$. Domains $\Omega_{n}$, $n=0,1,2, \ldots$ are defined by $\Omega_{0}=\mathbf{R}^{2}$ and $\Omega_{n}=B_{\varepsilon}\left(\mathbf{x}_{n}\right)$ for $n \geqq 1$. Identification maps $J_{n}: L^{2}\left(\Omega_{n}\right) \rightarrow L^{2}\left(\mathbf{R}^{2}\right)$ are defined by:

$$
\left(J_{n} \phi\right)(\mathbf{x})=g_{n}(\mathbf{x}) j_{n}(\mathbf{x}) \phi(\mathbf{x}), \quad \text { for } \quad \phi \in L^{2}\left(\Omega_{n}\right),
$$

where $g_{0}(\mathbf{x})=1$, and

$$
J\left(\bigoplus_{n \geqq 0} \phi_{n}\right)(\mathbf{x})=\sum_{n \geqq 0}\left(J_{n} \phi_{n}\right)(\mathbf{x}) \text { for } \bigoplus_{n \geqq 0} \phi_{n} \in \underset{n \geqq 0}{\bigoplus} L^{2}\left(\Omega_{n}\right) .
$$

We will denote $\bigoplus_{n \geqq 0} L^{2}\left(\Omega_{n}\right)$ by $\mathscr{H}_{0}$. 
Hamiltonian for the individual atomic systems is defined as follows: for $n \geqq 1$, $h_{n}$ is defined by:

$$
h_{n}=-\left(\partial / \partial \mathbf{x}-\mathbf{i} \mathbf{A}_{n}(\mathbf{x})\right)^{2}+\mu \mathscr{V}_{n}(\mathbf{x}), \text { on } L^{2}\left(\Omega_{n}\right),
$$

with Dirichlet boundary conditions, where $\mathscr{V}_{n}(\mathbf{x})=\mathscr{V}\left(\mathbf{x}-\mathbf{x}_{n}\right)$ and $\mathbf{A}_{n}(\mathbf{x})=$ $\left(-B\left(x_{2}-x_{n, 2}\right) / 2, B\left(x_{1}-x_{n, 1}\right) / 2\right)$. Let $\delta_{0}=\operatorname{Min}\left(\delta\right.$, inf $\left.\left\{\mathscr{V}(x) ; x \notin B_{\varepsilon^{\prime}}(0)\right\}\right)$ where $\delta$ is the constant in Assumptions (A), and we define $\mathscr{V}_{0}(x)=\operatorname{Max}\left(\delta_{0}, V(x)\right)$.

$h_{0}$ is defined by:

$$
h_{0}=-(\partial / \partial \mathbf{x}-\mathbf{i} \mathbf{A}(\mathbf{x}))^{2}+\mu \mathscr{V}_{0}(\mathbf{x}), \text { on } L^{2}\left(\Omega_{0}\right),
$$

and $H_{0}$ on $\mathscr{H}_{0}$ by $H_{0}=\bigoplus_{n \geqq 0} h_{n}$.

Lemma 4.1. $J^{*}$ is an isometry from $\mathscr{H}$ to $\mathscr{H}_{0}$.

Proof. By definition we have:

$$
J^{*} \phi(\mathbf{x})=j_{0}(\mathbf{x}) \phi(\mathbf{x}) \oplus \underset{n \geqq 1}{\bigoplus_{n}} g_{n}(\mathbf{x})^{*} j_{n}(\mathbf{x}) \phi(\mathbf{x}) .
$$

Hence

$$
\begin{aligned}
\left\|J^{*} \phi\right\|^{2} & =\int_{\mathbf{x} \in \mathbf{R}^{2}}\left|j_{0}(\mathbf{x}) \phi(\mathbf{x})\right|^{2} d^{2} \mathbf{x}+\sum_{n \geqq 1} \int_{\mathbf{x} \in \mathbf{R}^{2}}\left|j_{n}(\mathbf{x}) \phi(\mathbf{x})\right|^{2} d^{2} \mathbf{x}, \\
& =\int_{\mathbf{x} \in \mathbf{R}^{2}}\left(\left|j_{0}(\mathbf{x})\right|^{2}+\sum_{n \geqq 1}\left|j_{n}(\mathbf{x})\right|^{2}\right)|\phi(\mathbf{x})|^{2} d^{2} \mathbf{x}=\|\phi\|^{2},
\end{aligned}
$$

because $\left|j_{0}(\mathbf{x})\right|^{2}=\prod_{n=1}^{\infty}\left|j_{n}^{\prime}(\mathbf{x})\right|^{2}=\prod_{n=1}^{\infty}\left(1-\left|j_{n}(\mathbf{x})\right|^{2}\right)=1-\sum_{n \geqq 1}\left|j_{n}(\mathbf{x})\right|^{2}$.

$J$ is a "non-commutative partition of the unity" and the error term of the approximation is given by the following Lemma:

Lemma 4.2. $J$ maps $D\left(H_{0}\right)$ into $D(H)$ and:

$$
(H-z)^{-1}=J\left(H_{0}-z\right)^{-1} J^{*}-(H-z)^{-1} M\left(H_{0}-z\right)^{-1} J^{*},
$$

where $M:=H J-J H_{0} \in B\left(D\left(H_{0}\right), \mathscr{H}\right) . M$ is decomposed as $\bigoplus_{n \geqq 0} m_{n}$ with:

$$
m_{n}=H J_{n}-J_{n} h_{n}=-g_{n}(\mathbf{x})\left\{\left(\partial / \partial \mathbf{x}-\mathbf{i} \mathbf{A}_{n}\right)\left(\partial j_{n} / \partial \mathbf{x}\right)+\left(\partial j_{n} / \partial \mathbf{x}\right)\left(\partial / \partial \mathbf{x}-\mathbf{i} \mathbf{A}_{n}\right)\right],
$$

where we set $\mathbf{A}_{0}=\mathbf{A}(\mathbf{x})$.

Lemma 4.2 follows by easy computation. Notice that the $m_{n}$ 's $n \geqq 1$, are unitarily equivalent up to a magnetic translation.

\section{Operator Algebras and Connes's Formula}

In this section, we review definitions and basic properties of some algebras we consider. Again we fix $V=V_{\omega}$ throughout this section.

(a) *-Algebras on $\left.\mathscr{H}=L^{2}(\mathbf{R})\right)^{2}: a \in B\left(L^{2}\left(\mathbf{R}^{2}\right)\right)$ is an element of $\mathscr{A}_{0}$ if it has an integral kernel $k(\mathbf{x}, \mathbf{y})$ which is bounded, continuous and such that $k(\mathbf{x}, \mathbf{y})=0$ if $|\mathbf{x}-\mathbf{y}| \geqq C$ for some $C>0$. Let $\mathscr{A}$ be the $C^{*}$-Algebra generated by $\mathscr{A}_{0}$. 
(b) $\mathbf{Z}_{2}$-graded representation: Following [27,6], we introduce a trivially $\mathbf{Z}_{2}$-graded algebra as follows: let $\mathscr{H}^{\sim}=\mathscr{H}_{+} \oplus \mathscr{H}_{-}$, where $\mathscr{H}_{+}=\mathscr{H}_{-}=\mathscr{H}^{2} L^{2}\left(\mathbf{R}^{2}\right)$, and define a degree operator $G$ and a Dirac phase $F$ by:

$$
G=\left[\begin{array}{rr}
1 & 0 \\
0 & -1
\end{array}\right] ; \quad F=\left[\begin{array}{ll}
0 & \bar{D} \\
D & 0
\end{array}\right]
$$

with $D=\left(x_{1}+\mathbf{i} x_{2}\right) /|\mathbf{x}|$ for $\mathbf{x} \in \mathbf{R}^{2}$. We identify $\mathscr{A}$ with the set of diagonal elements in $B\left(\mathscr{H}^{\sim}\right): a \rightarrow a \oplus a \in B\left(\mathscr{H}^{\sim}\right)$. A "differential" $d: B\left(\mathscr{H}^{\sim}\right) \rightarrow B\left(\mathscr{H}^{\sim}\right)$ is given by

$$
d T=\mathbf{i}\left\{F T-(-)^{\operatorname{deg}(T)} T F\right\},
$$

for $T$ of homogeneous degree (i.e. $\left.G T=(-)^{\operatorname{deg}(T)} T G\right)$, and extended to $B\left(\mathscr{H}^{\sim}\right)$ by linearity. Since the representation of $\mathscr{A}$ is included in the subalgebra of degree zero, $d a=\mathrm{i}[F, a]$ for $a \in \mathscr{A}$.

(c) p-summable Fredholm module: Let $\mathscr{L}^{p}\left(\mathscr{H}^{\sim}\right)$ be the Schatten (or trace) ideal of order $p$ (i.e. $T \in \mathscr{L}^{p}\left(\mathscr{H}^{\sim}\right)$ whenever $\left.\|T\|_{\mathscr{L}_{p}}:=\left\{\operatorname{Tr}\left(\left|T^{*} T\right|^{p / 2}\right)\right\}^{2 / p}<\infty\right)$. An element $a \in \mathscr{A}$ is called $p$-summable whenever $d a=\mathrm{i}[F, a] \in \mathscr{L}^{p}\left(\mathscr{H}^{\sim}\right)$. A subalgebra $\mathscr{A}^{\prime}$ of $\mathscr{A}$ is called $p$-summable if all its elements are $p$-summable. In particular, the set of all $p$-summable elements of $\mathscr{A}$ forms a ${ }^{*}$-subalgebra of $\mathscr{A}$ which is denoted by $\mathscr{A}^{(p)}$. The representation of $\mathscr{A}$ on $\mathscr{H}^{\sim}$ is called $p$-summable Fredholm module over $\mathscr{A}^{\prime}$.

Lemma 5.1. Let $P=E_{I}(H)$ be an eigenprojection of $H$ with $I$ being an isolated compact subset of $\sigma(H)$. Then $P$ is $p$-summable for any $p>2$.

Lemma 5.1 will be proved in Appendix A.

(d) The Chern class: A 2-cocycle $\tau_{2}$ on $\mathscr{A}^{(3)}$ is defined by:

$$
\tau_{2}\left(a_{0}, a_{1}, a_{2}\right)=-(1 / 2) \operatorname{Tr}\left\{G F\left[F, a_{0}\right]\left[F, a_{1}\right]\left[F, a_{2}\right]\right\},
$$

for $a_{0}, a_{1}, a_{2} \in \mathscr{A}^{(3)}$, where $\operatorname{Tr}$ is the usual trace on $\mathscr{H}^{\sim}$. The (second) Chern class of projection $e \in \mathscr{A}^{(3)}$ is defined by

$$
\operatorname{Ch}(e)=\tau_{2}(e, e, e)=-1 / 2 \operatorname{Tr}\left\{G F[F, e]^{3}\right\} .
$$

$\mathrm{Ch}(\cdot)$ satisfies the following properties: (i) invariance: if $e$ and $f$ are equivalent projections, namely if there is $u \in \mathscr{A}^{(3)}$ such that $u u^{*}=e$ and $u^{*} u=f$ then $\mathrm{Ch}(e)=\mathrm{Ch}(f)$; (ii) additivity: if $e$ and $f$ are orthogonal, namely if $e f=f e=0$, then $\mathrm{Ch}(e+f)=\mathrm{Ch}(e)+\mathrm{Ch}(f)$; (iii) $\mathrm{Ch}(e)$ is an integer for any projection $e \in \mathscr{A}^{(3)}$ $[29,27]$

(e) Algebra on $\mathscr{H}_{0}$ : Analogously to (a) we define the algebras $\mathscr{B}_{0}$ and $\mathscr{B}$ on $\mathscr{H}_{0}=\bigoplus_{n \geq 0} L^{2}\left(\Omega_{n}\right)$ as follows: $b \in B\left(\mathscr{H}_{0}\right)$ is an element of $\mathscr{B}_{0}$ if every matrix element $b_{i j} \in B\left(L^{2}\left(\Omega_{j}\right), L^{2}\left(\Omega_{i}\right)\right)$ has a uniformly bounded, continuous integral kernel $k_{i j}(\mathbf{x}, \mathbf{y})$ such that $k_{i j}(\mathbf{x}, \mathbf{y})=0$ whenever $|\mathbf{x}-\mathbf{y}| \geqslant C$ for some $(i, j)$-independent constant $C>0$. Let $\mathscr{B}$ be the $C^{*}$-Algebra generated by $\mathscr{B}_{0}$.

(f) $\mathrm{Z}_{2}$-graded representation, $p$-summable fredholm module, Chern clàss on $\mathscr{B}$ : the trivially $\mathbf{Z}_{2}$-graded representation is constructed in exactly the same way as in (b) on the space $\mathscr{H}_{0}=\mathscr{H}_{0+} \oplus \mathscr{H}_{0-}$, where $\mathscr{H}_{0+}=\mathscr{H}_{0-}=\mathscr{H}_{0}=\bigoplus_{n \geqq 0} L^{2}\left(\Omega_{n}\right)$. $p$-summability for the Chern class is defined analogously on $\mathscr{H}_{0}^{\tilde{a}}$ and satisfies the same properties. In Particular, $\mathscr{B}^{(p)}$ is the subalgebra of $p$-summable elements of 
$\mathscr{B}$, and $\tau_{2}$ is defined by (5.3) in much the same way. Analogously to Lemma 5.1 we obtain the following result (see Appendix A):

Lemma 5.2. The statements of Lemma 5.1 hold for $H_{0}$ instead of $H$.

Since $J^{\sim *}:=J^{*} \oplus J^{*}$ is an isometry from $\mathscr{H}^{\sim}$ to $\mathscr{H}_{0}^{\sim}$, and since $J^{\sim *}$ commutes with both $G$ and $D$, the next lemma follows immediately:

Lemma 5.3. Suppose $a_{i} \in \mathscr{A}^{(3)}, i=0,1,2$, then $J^{\sim *} a_{i} J^{\sim} \in \mathscr{B}^{(3)}$ and:

$$
\tau_{2}\left(a_{0}, a_{1}, a_{2}\right)=\tau_{2}\left(J^{\sim *} a_{0} J^{\sim}, J^{\sim *} a_{1} J^{\sim}, J^{\sim *} a_{2} J^{\sim}\right) .
$$

In particular, for any projection $e \in \mathscr{A}^{(3)}, J^{\sim *} e J^{\sim}$ is a projection of $\mathscr{B}^{(3)}$ which satisfies $\mathrm{Ch}\left(J^{\sim *} e J^{\sim}\right)=\mathrm{Ch}(e)$.

Finally we present Connes formula, which relates the 2-character $\tau_{2}$ defined by (5.3) with the one defined by (3.13) [27, part I, Sect. 9]. Let us set $X=\{a \in \mathcal{O}$; $\left.\int_{\omega \in \Omega} \mathbf{P}(d \omega)\left\{\left\|a(\omega, \cdot)\langle\cdot\rangle^{2+\eta}\right\|_{L^{3 / 2}}\right\}^{3}<\infty\right\}$ for some $\eta>0$.

Theorem 5.4 (Connes formula).

Let $A_{0} \in X$ and $A_{i} \in X \cap \mathscr{H}_{1}, i=1,2$. Then $\pi_{\omega}\left(A_{i}\right)$ is 3-summable for a.e. $\omega \in \Omega$ and

$$
\tau_{2}\left(A_{0}, A_{1}, A_{2}\right)=\int_{\omega \in \Omega} \mathbf{P}(d \omega) \tau_{2}\left(\pi_{\omega}\left(A_{0}\right), \pi_{\omega}\left(A_{1}\right), \pi_{\omega}\left(A_{2}\right)\right) .
$$
Corollary $5.5[6,7]$. If $F$ belongs to a gap of $\bigcup_{\omega \in \Omega} \sigma\left(H_{\omega}\right)$, the Hall conductance is
given by:

$$
\sigma_{H}=\operatorname{Ch}\left(P_{F, \omega}\right) \text { for a.e. } \omega \in \Omega \text {. }
$$

The Corollary follows from Theorem 5.4 and the properties that $\mathrm{Ch}\left(P_{F, \omega}\right)$ is almost surely constant (see[5]). Theorem 5.5 will be proved in Appendix C. Now it is sufficient to prove that $\operatorname{Ch}\left(P_{F, \omega}\right)=0$ almost surely to show that the Hall conductance vanishes.

\section{Vanishing of the Chern Class of Eigenprojections}

Here we consider the Chern class of certain eigenprojections of $H$ and $H_{0}$, and we prove that they are zero if $\mu$ is large enough.

Let $\mathbf{d}$ be the Agmon distance between 0 and $\partial B_{\varepsilon^{\prime}}(0)$ with respect to $\mathscr{V}$, i.e. $\mathbf{d}=\inf \int_{0}^{1} V(\gamma(t))^{1 / 2} d \gamma(t)$, where $\gamma$ runs over the set of piecewise differentiable, continuous paths with $\gamma(0)=0$ and $\gamma(1) \in \partial B_{\varepsilon^{\prime}}(0)$. Let $\left\{E_{k}(\mu)\right\}_{k=1, \ldots, N}$ be a finite set of eigenvalues of $h$ satisfying the following conditions:

Assumption (b). For each $k, E_{k}(\mu) / \mu \rightarrow 0$ as $\mu \rightarrow \infty$. Moreover, for some $\eta>0$,

$$
\operatorname{dist}\left(\left\{E_{k}(\mu)\right\}, \quad \sigma(h) \backslash\left\{E_{k}(\mu)\right\}\right) \geqq \exp \left(-\mu^{1 / 2}(\mathbf{d}-\eta)\right) .
$$

As noticed in Sect. 2, each eigenvalue has an asymptotic expansion $\mu^{1 / 4}$ as $\mu \rightarrow \infty$. So the set of eigenvalues having the same leading terms satisfies assumption $(B)$, but our assumption includes cases with complicated degeneracy. 
For simplicity, we suppose $\sigma(h) \cap\left[E_{-}, E_{+}\right]=\left\{E_{k}(\mu)\right\}$ with $E_{-}=\inf \left\{E_{k}(\mu)\right\}$, $E_{+}=\sup \left\{E_{k}(\mu)\right\}$, without loss of generality. Let $\beta=1 / 2 \exp \left(-\mu^{1 / 2}(\mathbf{d}-\eta)\right)$, $I=\left[E_{-}-\beta, E_{+}+\beta\right], P=E_{I}(H), P_{0}=E_{I}\left(H_{0}\right)$. Our main technical results are:

Theorem 6.1. Suppose Assumptions (A). If $E=E(\mu)$ satisfies $E / \mu \rightarrow 0$ as $\mu \rightarrow \infty$ and

$$
\operatorname{dist}(E, \sigma(h)) \geqq \exp \left(-\mu^{1 / 2}(\mathbf{d}-\eta)\right)
$$

for some $\eta>0$, then $E \in \rho\left(H_{\omega}\right)$ for almost all $\omega \in \Omega$ and all $\mu$ 's large enough. $\diamond$ In particular, we have $E_{ \pm} \in \rho\left(H_{\omega}\right)$ almost surely.

Theorem 6.2. Suppose Assumptions $(A) \&(B)$, and let $P$ and $P_{0}$ be defined as above. Then for any $\eta>0$, there is $C>0$ such that:

$$
\left\|J^{*} P J-P_{0}\right\| \leqq C \exp \left(-\mu^{1 / 2}(\mathbf{d}-\eta)\right) . \diamond
$$

We will prove Theorems 6.1 and 6.2 in Sect. 7. We will consider the case $\mathscr{V}=\mathscr{V}_{\omega}$ satisfying Assumptions (A), and we will drop the expression "almost surely."

Lemma 6.3. $\mathrm{Ch}(P)=\mathrm{Ch}\left(P_{0}\right)$ if $\mu$ is sufficiently large.

Proof. By Lemma 5.3, Ch $(\mathrm{P})=\mathrm{Ch}\left(J^{*} P J\right)$. Since $\left\|J^{*} P J-P_{0}\right\|<1$ for large $\mu$ by Theorem 6.2, $J^{*} P J$ and $P_{0}$ are unitarily equivalent (Lemma D.1 in Appendix D). The invariance property of $\mathrm{Ch}(\cdot)$ gives the result.

Lemma 6.4. $\mathrm{Ch}\left(P_{0}\right)=0$ if $\mu$ is sufficiently large.

Proof. D leaves the decomposition of $H_{0}$ invariant; using the additivity of $\mathrm{Ch}(\cdot)$, we have:

$$
\operatorname{Ch}\left(P_{0}\right)=\sum_{n \geqq 0} \operatorname{Ch}\left(P_{0, n}\right),
$$

with $P_{0, n}=E_{I}\left(h_{n}\right)$ and $\mathrm{Ch}(\cdot)$ is defined on $L^{2}\left(\Omega_{n}\right)$ by the same equation. Since $\left[\mu \delta_{0}, \infty\right) \supset \sigma\left(h_{0}\right)$, we obtain $P_{0,0}=0$ for large $\mu$. Since $P_{0, n}$ is finite dimensional, we easily get:

$$
\operatorname{Tr}\left(G F\left[F, P_{0, n}\right]^{3}\right)=2\left\{\operatorname{Tr}\left(G P_{0, n} F P_{0, n} F\right)-\operatorname{Tr}\left(G P_{0, n}\right)\right\},
$$

where $\operatorname{Tr}$ is the trace on $L^{2}\left(\Omega_{n}\right) \oplus L^{2}\left(\Omega_{n}\right)$. We denote by tr the trace on $L^{2}\left(\Omega_{n}\right)$. Then:

$$
\begin{aligned}
\operatorname{Tr}\left(G P_{0, n}\right) & =\operatorname{tr}\left(P_{0, n}\right)-\operatorname{tr}\left(P_{0, n}\right)=0, \\
\operatorname{Tr}\left(G P_{0, n} F P_{0, n} F\right) & =\operatorname{tr}\left(P_{0, n} D^{*} P_{0, n} D\right)-\operatorname{tr}\left(P_{0, n} D P_{0, n} D^{*}\right)=0,
\end{aligned}
$$

This implies $\mathrm{Ch}\left(P_{0, n}\right)=0$, concluding the proof.

Proof of Theorem 2.1. At first we set $a=\mathbf{d}-\eta$, with $0<\eta<\mathbf{d}$. Then the set of eigenvalues of $h:\left\{E_{k} ; E_{k}<F\right\}$ satisfies assumption (B), and by Theorem 6.1, $F$ lies in a gap of the spectrum of $H_{\omega}$ almost surely (namely for $\omega$ 's satisfying Assumptions (A)). By Lemmas 6.3 and 6.4, $\mathrm{Ch}\left(P_{F}\right)=0$ almost surely and $\sigma_{H}=0$ now follows from Corollary 5.5 of Connes formula.

\section{Proof of Theorems 6.1 and 6.2}

In this section we fix $\omega \in \Omega$ satisfying Assumptions (A), but the estimates are uniform in $\omega$. 
Lemma 7.1. Suppose $z=z(\mu) \in \mathrm{C}$ satisfies as $\mu \rightarrow \infty$ and for some $\eta>0$ :

$$
|\operatorname{Re}(z)| / \mu \rightarrow 0, \quad \operatorname{dist}(z, \sigma(h)) \geqq \exp \left(-\mu^{1 / 2}(\mathbf{d}-\eta)\right) .
$$

Then for any $\chi \in L^{\infty}\left(B_{\varepsilon}(0)\right)$ with support included in $B_{\varepsilon}(0) \backslash B_{\varepsilon^{\prime}}(0)$,

$$
\left\|\chi(h-z)^{-1}\right\| \leqq C \mu^{-1} ; \quad\left\|\chi(\partial / \partial \mathbf{x}-\mathbf{i A})(h-z)^{-1}\right\| \leqq C \mu^{-1 / 2} .
$$

These estimates are due to Briet, Combes and Duclos [30]. They proved estimates of this type in the case $\mathbf{A}=0$, but the proof works even in the presence of a magnetic field. The next lemma is an easy consequence of the definition of $h_{0}$ :

Lemma 7.2. Suppose $z=z(\mu) \in \mathbf{C}$ satisfies $|\operatorname{Re}(z)| / \mu \rightarrow 0$ as $\mu \rightarrow \infty$. Then for any $\chi \in L^{\infty}\left(\mathbf{R}^{2}\right)$,

$$
\begin{gathered}
\left\|\chi\left(h_{0}-z\right)^{-1}\right\| \leqq C \mu^{-1} \\
\left\|\chi(\partial / \partial \mathbf{x}-\mathbf{i A})\left(h_{0}-z\right)^{-1}\right\| \leqq C \mu^{-1 / 2}
\end{gathered}
$$

Now we estimate the error terms for the approximation of the resolvent in Lemma 4.2.

Lemma 7.3. For $z=z(\mu) \in \mathrm{C}$ like in Lemma 7.1,

$$
\left\|M\left(H_{0}-z\right)^{-1}\right\| \leqq C \mu^{-1 / 2} .
$$

Proof. At first, we note

$$
M\left(H_{0}-z\right)^{-1}=\bigoplus_{n \geqq 0} m_{n}\left(h_{n}-z\right)^{-1},
$$

by Lemma 4.2. From (4.8) and Lemma 7.2, it is easy to see that,

$$
\left\|m_{0}\left(h_{0}-z\right)^{-1}\right\| \leqq C \mu^{-1 / 2} .
$$

On the other hand, since $\partial j_{n} / \partial \mathbf{x}$ is supported by $B_{\varepsilon}\left(x_{n}\right) \backslash B_{\varepsilon^{\prime}}\left(x_{n}\right)$ we can apply Lemma 7.1 to get a constant $C>0$ such that for $n>1$,

$$
\left\|m_{n}\left(h_{n}-z\right)^{-1}\right\| \leqq C \mu^{-1 / 2} .
$$

Noting that the ranges of the $m_{n}$ 's are mutually orthogonal for $n \geqq 1$, because the supports of the $j_{n}$ 's are disjoint, we get:

$$
\begin{aligned}
&\left\|M\left(H_{0}-z\right)^{-1} \bigoplus_{n \geqq 0} \phi_{n}\right\|^{2} \\
&=\left\|m_{0}\left(h_{0}-z\right)^{-1} \phi_{0}\right\|^{2}+2 \sum_{n=1}^{\infty} \operatorname{Re}\left(m_{0}\left(h_{0}-z\right)^{-1} \phi_{0}, m_{n}\left(h_{n}-z\right)^{-1} \phi_{n}\right) \\
&+\sum_{n=1}^{\infty}\left\|m_{n}\left(h_{n}-z\right)^{-1} \phi_{n}\right\|^{2} \leqslant\left\|m_{0}\left(h_{0}-z\right)^{-1} \phi_{0}\right\|^{2} \\
&+2\left\{\sum_{n=1}^{\infty}\left\|\chi_{\Omega n} m_{0}\left(h_{0}-z\right)^{-1} \phi_{0}\right\|^{2}\right\}^{1 / 2}\left\{\sum_{n=1}^{\infty}\left\|m_{n}\left(h_{n}-z\right)^{-1} \phi_{n}\right\|^{2}\right\}^{1 / 2} \\
&+\sum_{n=1}^{\infty}\left\|m_{n}\left(j h_{n}-z\right)^{-1} \phi_{n}\right\|^{2} \leqq \sum_{n=0}^{\infty}\left\|m_{n}\left(h_{n}-z\right)^{-1} \phi_{n}\right\|^{2} \\
&+2\left\|m_{0}\left(h_{0}-z\right)^{-1} \phi_{0}\right\|\left\{\sum_{n=1}^{\infty}\left\|m_{n}\left(h_{n}-z\right)^{-1} \phi_{n}\right\|^{2}\right\}^{1 / 2},
\end{aligned}
$$


by Hölder's inequality. Now using (7.8) and (7.9), we obtain

$$
\begin{aligned}
\cdot\left\|M\left(H_{0}-z\right)^{-1} \bigoplus_{n \geqq 0} \phi_{n}\right\|^{2} \leqq & C \mu^{-1}\left\|\phi_{0}\right\|^{2}+\left\{C \mu^{-1}\left\|\phi_{0}\right\|^{2}\right\}^{1 / 2}\left\{C \mu^{-1} \sum_{n=1}^{\infty}\left\|\phi_{n}\right\|^{2}\right\}^{1 / 2} \\
& +C \mu^{-1} \sum_{n=1}^{\infty}\left\|\phi_{n}\right\|^{2} \leqq C \mu^{-1} \sum_{n=0}^{\infty}\left\|\phi_{n}\right\|^{2} . \diamond
\end{aligned}
$$

Lemma 7.4. For $z=z(\mu) \in \mathbf{C}$ like in Lemma 7.1, $z \in \rho(H)$ for $\mu$ large enough, and

$$
(H-z)^{-1}=J\left(H_{0}-z\right)^{-1} J^{*}\left(1+M\left(H_{0}-z\right)^{-1} J^{*}\right)^{-1} .
$$

Proof. (7.12) holds for $\operatorname{Re}(z)$ sufficiently negative, by Lemma 4.2, and it holds if the right-hand side is well-defined by analyticity. Then Lemma 7.4 follows from Lemma 7.3. Theorem 6.1 now follows from Lemma 7.4.

Lemma 7.5. For $z=z(\mu) \in \mathbf{C}$ like in Lemma 7.1.,

$$
\left\|\left(J^{*} J-1\right)\left(H_{0}-z\right)^{-1}\right\| \leqq C \mu^{-1} .
$$

Proof. Since $J_{n}^{*} J_{m}=0$ for $n, m \geqq 1$ and $n \neq m$, we have

$$
J^{*} J-\mathbf{1}=\bigoplus_{n \geqq 0}\left(J_{n}^{*} J_{n}-\mathbf{1}\right)+\bigoplus_{n \geqq 1} J_{0}^{*} J_{n}+\bigoplus_{n \geqq 1} J_{n}^{*} J_{0} .
$$

Since $\left(j_{n}^{2}-1\right)$ is supported in $B_{\varepsilon}\left(x_{n}\right) \backslash B_{\varepsilon^{\prime}}\left(x_{n}\right)$, we have

$$
\left\|\left(J_{n}^{*} J_{n}-1\right)\left(h_{n}-z\right)^{-1}\right\| \leqq C \mu^{-1},
$$

for $n \geqq 0$ by Lemmas 7.1 and 7.2. We have

$$
\left\|\bigoplus_{n \geqq 1} J_{n}^{*} J_{0}\left(h_{0}-z\right)^{-1} \phi\right\|^{2}=\sum_{n \geqq 1}\left\|j_{n} j_{0}\left(h_{0}-z\right)^{-1} \phi\right\|^{2}=\left\|\left(\sum_{n \geqq 1} j_{n} j_{0}\right)\left(h_{0}-z\right)^{-1} \phi\right\|^{2}
$$

because $\operatorname{supp}\left(j_{n}\right)$ are disjoint for $n \geqq 1$. Noting that $\left(\sum_{n \geqq 1} j_{n} j_{0}\right)<1$, we obtain

$$
\left\|\bigoplus_{n \geqq 1} J_{n}^{*} J_{0}\left(h_{0}-z\right)^{-1} \phi\right\|^{2} \leqq C \mu^{-1}
$$

Similarly,

$$
\begin{aligned}
\left\|\bigoplus_{n \geqq 1} J_{0}^{*} J_{n}\left(h_{n}-z\right)^{-1} \phi_{n}\right\|^{2} & =\sum_{n \geqq 1}\left\|J_{0}^{*} J_{n}\left(h_{n}-z\right)^{-1} \phi_{n}\right\|^{2}=\sum_{n \geqq 1}\left\|j_{0} j_{n}\left(h_{n}-z\right)^{-1} \phi_{n}\right\|^{2} \\
& \leqq \sup _{n \geqq 1}\left\|j_{0} j_{n}\left(h_{n}-z\right)^{-1}\right\| \sum_{n \geqq 1}\left\|\phi_{n}\right\|^{2} \leqq C \mu^{-2} \sum_{n \geqq 1}\left\|\phi_{n}\right\|^{2},
\end{aligned}
$$

because again $\left(j_{0} j_{n}\right)$ is supported in $B_{\varepsilon}\left(x_{n}\right) \backslash B_{\varepsilon^{\prime}}\left(x_{n}\right)$. Then (7.13) follows from (7.14)-(7.18). 
Proof of Theorem 6.2. Let $\left\{E_{k}\right\}_{k=1, \ldots, N}$ be the set of eigenvalues in Assumptions (A) and (B), and let $D$ be a neighbourhood of $\left\{E_{k}\right\}$ :

$$
D=\bigcup_{k}\left\{z \in \mathbf{C} ;\left|\operatorname{Re}(z)-E_{k}\right| \leqq \beta, \operatorname{Im}(z) \leqq \beta\right\},
$$

with $\beta=1 / 2 \exp \left(-\mu^{1 / 2}(\mathbf{d}-\eta)\right), 0<\eta<\mathbf{d}$, and we set $\Gamma=\partial D$. We notice that the points on $\Gamma$ satisfy uniformly. For sufficiently large $\mu$, we have

$$
J^{*} P J=-1 /(2 \mathrm{i} \pi) \int_{\Gamma} J^{*}(H-z)^{-1} J d z
$$

and by (4.7),

$$
\begin{aligned}
J^{*}(H-z)^{-1} J= & J^{*} J\left(H_{0}-z\right)^{-1} J^{*} J-J^{*}(H-z)^{-1} M J\left(H_{0}-z\right)^{-1} J^{*} J \\
= & \left(H_{0}-z\right)^{-1}+\left\{J^{*} J\left(H_{0}-z\right)^{-1} J^{*} J-\left(H_{0}-z\right)^{-1}\right\} \\
& +\left\{J^{*}(H-z)^{-1} J\right\}\left\{J^{*} M\left(H_{0}-z\right)^{-1} J^{*} J\right\} .
\end{aligned}
$$

Thus we obtain

$$
\begin{aligned}
J^{*}(H-z)^{-1} J= & \left(H_{0}-z\right)^{-1}(1+L)^{-1} \\
& +\left\{J^{*} J\left(H_{0}-z\right)^{-1} J^{*} J-\left(H_{0}-z\right)^{-1}\right\}(1+L)^{-1}
\end{aligned}
$$

with $L=-J^{*} M\left(H_{0}-z\right)^{-1} J^{*} J$. Substituting (7.22) to (7.20), we have

$$
\begin{aligned}
J^{*} P J= & -1 /(2 \mathbf{i} \pi) \int_{\Gamma}\left(H_{0}-z\right)^{-1}(\mathbf{1}+L)^{-1} d z \\
& -1 /(2 \mathbf{i} \pi) \int_{\Gamma}\left\{J^{*} J\left(H_{0}-z\right)^{-1} J^{*} J-\left(H_{0}-z\right)^{-1}\right\}(\mathbf{1}+L)^{-1} d z \\
= & P_{0}-1 /(2 \mathbf{i} \pi) \int_{\Gamma}\left(H_{0}-z\right)^{-1}\left\{(\mathbf{1}+L)^{-1}-1\right\} d z \\
& -1 /(2 \mathbf{i} \pi) \int_{\Gamma}\left\{\left(J^{*} J-\mathbf{1}\right)\left(H_{0}-z\right)^{-1}+J^{*} J\left(\left(J^{*} J-1\right)\left(H_{0}-z^{*}\right)^{-1}\right)^{*}\right\}(\mathbf{1}+L)^{-1} d z .
\end{aligned}
$$

The integrand of the second and the third terms are of order $\mu^{-1 / 2}$ and $\mu^{-1}$ by Lemmas 7.3 and 7.5 respectively. On the other hand, there is a constant $C>0$ uniform in $\mu$, such that for large $\mu$ 's the length of the path $\Gamma$ is dominated by

$$
|\Gamma| \leqq 8 \beta \text { \# }\{(\text { eigenvalues of } h) \leqq F\} \leqq C \beta F^{2} \leqq O\left(\mu^{2}\right) \exp \left(-\mu^{1 / 2}(\mathbf{d}-\eta)\right) \text {. }
$$

This complete the proof.

\section{Appendix A. p-Summability of Resolvents}

We consider the Schrödinger operator $H=-(\partial / \partial \mathbf{x}-\mathbf{i A}(\mathbf{x}))^{2}+V(\mathbf{x})$ on $L^{2}\left(\mathbf{R}^{2}\right)$ with $V \in L^{\infty}\left(\mathbf{R}^{2}\right)$ and $\mathbf{A}=\left(-B x_{2} / 2, B x_{1} / 2\right)$. Then $H$ is essentially self-adjoint on $\mathscr{C}_{0}^{\infty}\left(\mathbf{R}^{2}\right)$ [21, Chap. 10]. The purpose of this appendix is to prove the next proposition:

Proposition A.1. For $z \in \rho(H),(H-z)^{-1}$ is a p-summable element of $\mathscr{A}$ for any $p>2$. Moreover the $p^{\text {th }}$ trace norm of $\left[F,(H-z)^{-1}\right]$ is locally bounded in $\rho(H)$. 
Proposition A.2. For $z \in \rho\left(H_{0}\right),\left(H_{0}-z\right)^{-1}$ is a p-summable element of $\mathscr{B}$ for any $p>2$. Moreover the $p^{\text {th }}$ trace norm of $\left[F,\left(H_{0}-z\right)^{-1}\right]$ is locally bounded in $\rho(H)$.

Lemma 5.1 and 5.2 are direct consequences of Propositions A.1 and A.2 respectively. We notice that, as can be seen from the proof, the estimates depend only upon $\|V\|_{L^{\infty}}$ and $\operatorname{dist}(z, \sigma(H))$.

Proposition A.1 follows from the next proposition and lemma:

Proposition A.3. For $z \in \rho(H),(H-z)^{-1}$ has an integral kernel $k(\mathbf{x}, \mathbf{y})$ such that for any $1 \leqq p<\infty, \sup _{\mathbf{x}}\|k(\mathbf{x}, \cdot)\|_{L^{p}}<\infty$, sup $\|k(\cdot, \mathbf{y})\|_{L^{p}}<\infty$. Moreover, $k(\mathbf{x}, \mathbf{y})$ is continuous on $\mathbf{R}^{\mathbf{4}} \backslash\{\mathbf{x}=\mathbf{y}\}$ and for some $\alpha>0$, and for any $\delta>0$, there is a positive constant $C_{\delta}$ such that $|k(\mathbf{x}, \mathbf{y})| \leqq C_{\delta} \exp (-\alpha|\mathbf{x}-\mathbf{y}|)$ whenever $|\mathbf{x}-\mathbf{y}|>\delta$.

Lemma A.4. Let $A$ be an integral operator with a kernel $k(\mathbf{x}, \mathbf{y})$. If $k$ satisfies:

$$
\sup _{\mathbf{x}}\left\|\langle\mathbf{x}-\cdot\rangle^{m} k(\mathbf{x}, \cdot)\right\|_{L^{q}}<\infty,
$$

with $p>2,1 / p+1 / q=1, m>2 /(p-2)$, then $A$ is $p$-summable.

Here we have used the notation $\langle\mathbf{x}\rangle=\left(1+|\mathbf{x}|^{2}\right)^{1 / 2}$.

Proof of Lemma A.4. By definition, it is sufficient to show that the operator defined by the integral kernel $k(\mathbf{x}, \mathbf{y})(\mathbf{x} / \mathbf{x}|-\mathbf{y} /| \mathbf{y} \mid)$ belongs to the $p^{\text {th }}$ trace class. We set $\gamma>0$ so that $2 / m p<\gamma<1-2 / p$. By elementary calculations, if $\langle\mathbf{x}-\mathbf{y}\rangle \leqq\langle\mathbf{x}\rangle^{\gamma}$ then $|\mathbf{x} /| \mathbf{x}|-\mathbf{y} /| \mathbf{y} \| \leqq C\langle\mathbf{x}\rangle^{-(1-\gamma)}$. Hence we have:

$$
\begin{aligned}
& \int_{\mathbf{y} \in \mathbf{R} 2} d^{2} \mathbf{y}|k(\mathbf{x}, \mathbf{y})(\mathbf{x} /|\mathbf{x}|-\mathbf{y} /|\mathbf{y}|)|^{q} \\
& \quad \leqq C \int_{\langle\mathbf{x}-\mathbf{y}\rangle \leqq\langle\mathbf{x}\rangle^{r}} d^{2} \mathbf{y}|\mathbf{k}(\mathbf{x}, \mathbf{y})|^{q}\langle\mathbf{x}\rangle^{-(1-\gamma) q} \\
& +\int_{\langle\mathbf{x}-\mathbf{y}\rangle \geqq\langle\mathbf{x}\rangle^{\prime}} d^{2} \mathbf{y} \mid k(\mathbf{x}, \mathbf{y})\langle\mathbf{x}-\mathbf{y}\rangle^{m \mid q}\langle\mathbf{x}-\mathbf{y}\rangle^{-m q} \\
& \quad \leqq C\left\{\|k(\mathbf{x}, \cdot)\|_{L^{q}} q\langle\mathbf{x}\rangle^{-(1-\gamma) q}+\left\|\langle\mathbf{x}-\cdot\rangle^{m} k(\mathbf{x}, \cdot)\right\|_{L^{q}} q\langle\mathbf{x}\rangle^{-m \gamma q}\right\} \\
& \quad \leqslant C \operatorname{Max}\left\{\langle\mathbf{x}\rangle^{-(1-\gamma)},\langle\mathbf{x}\rangle^{-m \gamma}\right\}^{q} .
\end{aligned}
$$

Since $(1-\gamma) p>2$ and $\gamma m p>2$, we obtain

$$
\left.\left.\int_{\mathbf{x} \in \mathbf{R}^{2}} d^{2} \mathbf{x}\left|\int_{\mathbf{y} \in \mathbf{R}^{2}} d^{2} \mathbf{y}\right| k(\mathbf{x}, \mathbf{y})(\mathbf{x} /|\mathbf{x}|-\mathbf{y} /|\mathbf{y}|)\right|^{q}\right|^{p / q} \leqq C \int_{\mathbf{x} \in \mathbf{R}^{2}} d^{2} \mathbf{x}\langle\mathbf{x}\rangle^{-\operatorname{Min}((1-\gamma) p, \gamma m p)}<\infty .
$$

By Russo's Theorem [31], we conclude that the operator belongs to the $p^{\text {th }}$ trace class.

Proof of Proposition A.2. Since the operator $[D, \cdot]$ leaves the decomposition $\bigoplus_{n \geqq 0} L^{2}\left(\Omega_{n}\right)$ invariant, if suffices to prove the statement for $h_{0}$ and $\bigoplus_{n \geqq 1} h_{n}$ respectively. As for $h_{0}$ we can apply Proposition A.1. The resolvent $\bigoplus_{n \geqq 1}\left(h_{n}-z\right)^{-1}$ has an integral kernel $\bigoplus_{n \geqq 1} k_{n}(\mathbf{x}, \mathbf{y}), \mathbf{x}, \mathbf{y} \in \Omega_{n}$, and $K(\mathbf{x}, \mathbf{y})=\sum_{n \geqq 1} k_{n}(\mathbf{x}, \mathbf{y})$ can be considered as a kernel 
on $\mathbf{R}^{2}$ because the $\Omega_{n}$ 's are disjoints for $n \geqq 1$. Moreover $\left|k_{n}(\mathbf{x}, \mathbf{y})\right|=\left|k\left(\mathbf{x}-\mathbf{x}_{n}, \mathbf{y}-\mathbf{y}_{n}\right)\right|$, where $k$ is the integral kernel of $(h-z)^{-1}$ on $L^{2}\left(B_{\varepsilon}(0)\right)$. The operator $(h-z)^{-1}$ actually maps $\mathscr{H}_{0}^{-1-\eta}\left(B_{\varepsilon}(0)\right)$ into $\mathscr{H}^{1-\eta}\left(B_{\varepsilon}(0)\right)$ and the Dirac $\delta$-function belongs to $\mathscr{H}_{0}^{-1-\eta}\left(B_{\varepsilon}(0)\right)$ provided $0<\eta \leqq 1$; thus $k(\mathbf{x}, \cdot) \in \mathscr{H}^{1-\eta}\left(B_{\varepsilon}(0)\right)$, and hence $k\left(\mathbf{x},{ }^{\cdot}\right) \in L^{p}$ for any $p<\infty$ by the Sobolev embedding theorem. Thus $K$ satisfies (A.1) because $K(\mathbf{x}, \mathbf{y})=0$ whenever $|\mathbf{x}-\mathbf{y}|>2 \varepsilon$, and combining this with Lemma A.4, we finish the proof.

Now we prove Proposition A.3 by a series of lemmas (Lemmas A.5-A.7). We notice that such results are proved in [32] in a generalized form, but we give the proof here both for completeness and as a preparation for Appendix B. We may assume $\mathbf{x}=0$ (or $\mathbf{y}=0$ ) after changing the gauge if necessary, and we consider estimates near $\mathbf{x}=0$ (or $\mathbf{y}=0$ ) without loss of generality. We set

$$
L_{v}=-(\partial / \partial \mathbf{x}-\mathbf{i A}(\mathbf{x}))^{2}+2 v \cdot(\partial / \partial \mathbf{x}-\mathbf{i A}(\mathbf{x}))+V(\mathbf{x}),
$$

for $v \in \mathbf{C}^{2}$.

Lemma A.5. Let $\phi \in D\left(L_{v}\right)$, then $\phi$ is continuous and $|\phi(\mathbf{x})| \leqq C\left(\|\phi\|^{2}+\left\|L_{v} \phi\right\|^{2}\right)^{1 / 2}$ for $\mathbf{x} \in \mathbf{R}^{2}$.

Proof. Since $(\partial / \partial \mathbf{x}-\mathbf{i A}(\mathbf{x}))$ is infinitesimally $H$-bounded, $D\left(L_{v}\right)=D(H)$. As is easily seen,

$$
|\phi(0)| \leqq C\left(\|\phi\|_{L^{2}(B)^{2}}+\|\Delta \phi\|_{L^{2}(B)^{2}}\right)^{1 / 2} \leqq C\left(\|\phi\|_{L^{2}(B)^{2}}+\|H \phi\|_{L^{2}(B)^{2}}\right)^{1 / 2},
$$

with $B=\{\mathbf{x} ;|\mathbf{x}| \leqq 1\}$. Since $\phi$ is locally $\mathscr{H}^{2}$, it is Hölder continuous.

Lemma A.6. Let $z \in \rho\left(L_{v}\right)$, then $\left(L_{v}-z\right)^{-1}$ has an integral kernel $k_{v}(\mathbf{x}, \mathbf{y})$ satisfying (i) $\sup \left\|k_{v}(\mathbf{x}, \cdot)\right\|_{L^{p}(B)}<\infty$, sup $\left\|k_{v}(\cdot, \mathbf{y})\right\|_{L^{p}(B)}<\infty$ for any $1 \leqq p<\infty$, with $B=\stackrel{\mathbf{x}}{B_{1}}(0)$.

(ii) $k_{v}(\mathbf{x}, \mathbf{y})$ is continuous if $\mathbf{x} \neq \mathbf{y}$ and uniformly bounded on $\{(\mathbf{x}, \mathbf{y}) ;|\mathbf{x}-\mathbf{y}|>\delta\}$ for any $\delta>0$.

Proof. Since the Dirac function at $\mathbf{y}, \delta_{\mathbf{y}}$ belongs to $\mathscr{H}_{c}^{-1-\gamma}, k_{v}(\cdot, \mathbf{y})=\left(L_{v}-z\right)^{-1} \delta_{\mathbf{y}}$ belongs to $\mathscr{H}_{\text {loc }}^{1-\gamma}$ for $0<\gamma \leqq 1$, hence $k_{v}(\cdot, y) \in L_{\text {loc }}^{p}$ for $p<\infty$. Since the estimate is uniform in $\mathbf{y}$, we obtain the latter assertion of (i). The former is proved similarly.

Let $\chi(\mathbf{x})$ be a smooth function such that $\chi=0$ near 0 and $\chi=1$ for $|\mathbf{x}|>\delta>0$. It is easy to see that $\left(L_{v}-z\right) \chi\left(L_{v}-z\right)^{-1} \delta_{0} \in L^{2}$. This implies $\chi k_{v}(\cdot, 0) \in D\left(L_{v}\right)$, hence the assertion (ii) follows from Lemma A.5, and the homogeneity property.

Lemma A.7. Let $z \in \rho(H)$, then there is $\tau>0$ and for any $\gamma>0$, there is $C_{\gamma}>0$ such that

$$
|k(\mathbf{x}, \mathbf{y})| \leqq C_{\gamma} \exp (-\tau|\mathbf{x}-\mathbf{y}|) \quad \text { if } \quad|\mathbf{x}-\mathbf{y}| \geqq \gamma . \diamond
$$

Proof. Since $L_{v}=e^{i v \cdot \mathbf{x}} H e^{-i v \cdot \mathbf{x}}$,

$$
k_{v}(\mathbf{x}, \mathbf{y})=e^{i v \cdot(\mathbf{x}-\mathbf{y})} k_{0}(\mathbf{x}, \mathbf{y}),
$$

whenever $z \in \rho\left(L_{v}\right) \cap \rho(H)$. For $|v|$ small enough and $z \in \rho(H)$, then $z \in \rho\left(L_{v}\right)$ because 
$(\partial / \partial \mathbf{x}-\mathbf{i A})$ is $H$-bounded. Thus combining this with Lemma A.6, we obtain

$$
e^{\mid \operatorname{lm}(v \cdot(\mathbf{x}-\mathbf{y}) \mid}\left|k_{0}(\mathbf{x}, \mathbf{y})\right| \leqq C_{\gamma} \quad \text { if } \quad|\mathbf{x}-\mathbf{y}| \geqq \gamma .
$$

Since (A.8) holds for any small enough $v$, this implies (A.6).

We also need the following result in Sect. 3 (see also Eq. (2.5)):

Lemma A.8. For $z \in \rho(H),\left[\mathbf{X},(H-z)^{-1}\right]$ has a continuous integral kernel $(\mathbf{x}-\mathbf{y}) k(\mathbf{x}, \mathbf{y})$ and satisfies

$$
|(\mathbf{x}-\mathbf{y}) k(\mathbf{x}, \mathbf{y})| \leqq C \exp (-\tau|\mathbf{x}-\mathbf{y}|) \quad \text { if } \quad \mathbf{x}, \mathbf{y} \in \mathbf{R}^{2} .
$$

Proof. It is easy to see that $(\mathbf{x}-\mathbf{y}) k(\mathbf{x}, \mathbf{y})$ is the distribution kernel of $\left[\mathbf{X},(H-z)^{-1}\right]$. Moreover we have

$$
\begin{aligned}
(\mathbf{x}-\mathbf{y}) k(\mathbf{x}, \mathbf{y}) & =\left(\left[\mathbf{X},(H-z)^{-1}\right] \delta_{\mathbf{y}}\right)(\mathbf{x}) \\
& =-\left\{(H-z)^{-1}(\mathbf{x} \partial / \partial \mathbf{x}+\partial / \partial \mathbf{x} \cdot \mathbf{x})(H-z)^{-1} \delta_{\mathbf{y}}\right\}(\mathbf{x}),
\end{aligned}
$$

hence $(\cdot-\mathbf{y}) k(\cdot, \mathbf{y}) \in \mathscr{H}_{\text {loc }}^{2-\delta}\left(\mathbf{R}^{2}\right)$ which is contained in $\mathscr{C}\left(\mathbf{R}^{2}\right)$ for $0<\delta<1$. Combining this with Lemma A.7, we obtain (A.9).

Finally we note that the constant $C$ in (A.9) depend only upon $\|V\|_{\mathscr{L}_{\infty}}$ and $\operatorname{dist}(z, \sigma(H))$ as well as constants in other propositions.

$$
\text { Appendix B. Properties of } R_{\varepsilon}(z)=\left(1+\varepsilon H_{B}\right)^{-1}\left(H_{\omega}-z\right)^{-1}\left(1+\varepsilon H_{B}\right)^{-1}
$$

In this appendix we consider some properties of

$$
R_{\varepsilon}(z)=R_{\varepsilon}(\omega ; z)=\left(1+\varepsilon H_{B}\right)^{-1}\left(H_{\omega}-z\right)^{-1}\left(1+\varepsilon H_{B}\right)^{-1},
$$

with $z \in \bigcap_{\omega \in \Omega} \rho\left(H_{\omega}\right)$ and $\varepsilon>0$. Here $H_{B}=-(\partial / \partial \mathbf{x}-\mathbf{i A}(\mathbf{x}))^{2}$, and we will set $H_{0}=-\partial^{2} / \partial \mathbf{x}^{2}$.

First of all we will consider for $\Omega$ any closed subspace of the ball $\Omega_{m^{2}}$ of $L^{\infty}\left(\mathbf{R}^{2}\right)$ with radius $m^{2}$, endowed with the weak topology of $L^{1}\left(\mathbf{R}^{2}\right)$. In particular $\|V\|:=\sup \left\|V_{\omega}\right\|_{L^{\infty}} \leqq m^{2}$. Moreover by definition, for any element $f$ of $L^{1}\left(\mathbf{R}^{2}\right)$, the $\operatorname{map} \omega \in \Omega \rightarrow \int V_{\omega}(\mathbf{x}) f(\mathbf{x}) d^{2} \mathbf{x} \in \mathbf{C}$ is continuous. For simplicity we will write $V$ instead of $V_{\omega}$.

Proposition B.1. $R_{\varepsilon}(\omega ; z)$ has an integral kernel $k_{\varepsilon}(\omega ; \mathbf{x}, \mathbf{y})$ continuous with respect to $(\omega ; \mathbf{x}, \mathbf{y})$ vanishing as $|\mathbf{x}-\mathbf{y}| \rightarrow \infty$.

Proposition B.2. $R_{\varepsilon}(\omega ; z)$ converges to $\left(H_{\omega}-z\right)^{-1}$ as $\varepsilon \rightarrow 0$. In particular $R_{\varepsilon}(\omega ; z)$ and $\left(H_{\omega}-z\right)^{-1}$ are represented by elements of $\mathcal{O}$.

Before proving these propositions we will need the following results.

Lemma B.3. If $R(z)<-m^{2}$, the integral kernel $G_{B}(\mathbf{x}, \mathbf{y})$ of $\left(H_{B}-z\right)^{-1}$ is pointwise dominated by the integral kernel $G(\mathbf{x}-\mathbf{y})$ of $\left(H_{0}+m^{2}\right)^{-1}$.

Lemma B.4. Let $\mathscr{E}$ be a Banach space. Let $D$ be an open set in the complex plane C. Let $f: z \in D \rightarrow f(z) \in \mathscr{E}$ be a complex analytic function. We suppose that there is a 
subset $D_{0}$ of $D$ and a closed subspace $\mathscr{E}_{0}$ of $\mathscr{E}$ such that (i) $f(z) \in \mathscr{E}_{0}$ if $z \in D_{0}$, (ii) $D_{0}$ is a unicity domain for $D$, i.e. any holomorphic function on $D$ vanishing on $D_{0}$, vanishes on $D$. Then $f(z) \in \mathscr{E}_{0}$ for any $z \in D$.

Proof. For Lemma B.3 see [32, Sect. B.13]. The proof of Lemma B.4 goes as follows: suppose the conclusion fails. Then there is $z_{0} \in D$ such that $f\left(z_{0}\right) \notin \mathscr{E}_{0}$. By the Hahn-Banach theorem, there is a continuous linear form $x$ on $\mathscr{E}$ such that $x$ vanishes on $\mathscr{E}_{0}$, and that $\left\langle x, f\left(z_{0}\right)\right\rangle=1$. But the map $z \rightarrow\langle x, f(z)\rangle$ is analytic on $D$ and vanishes on $D_{0}$, a contradiction. $\diamond$

Lemma B.5. If $R^{(n)}(z)=\left(1+\varepsilon H_{B}\right)^{-1}\left(\left(H_{B}-z\right)^{-1} V_{\omega}\right)^{n}\left(H_{B}-z\right)^{-1}\left(1+\varepsilon H_{B}\right)^{-1}$ and if $\operatorname{Re}(z)<-m^{2}$, the integral kernel of $R^{(n)}(z)$ satisfies the estimate (for some $C>0$ ):

$$
\left|R^{(n)}(z)(\omega ; \mathbf{x}, \mathbf{y})\right| \leqq C\left\{\|V\| / m^{2}\right\}^{n} \exp (-m|\mathbf{x}-\mathbf{y}|) .
$$

Proof. Using Lemmas B.2 \& B.3, $\left|R^{(n)}(z)(\omega ; \mathbf{x}, \mathbf{y})\right|$ is pointwise dominated by:

$$
\left|R^{(n)}(z)(\omega ; \mathbf{x}, \mathbf{y})\right| \leqq\|V\|^{n}\left\{\left(H_{0}+m^{2}\right)^{-n-1}\left(\mathbf{1}+\varepsilon H_{0}\right)^{-2}\right\}(x-y),
$$

leading immediately to (B.2).

Proof of Proposition B.1. 1) Let us assume first that $\operatorname{Re}(z)<-m^{2}-\|V\|$. If $\|V\|<m^{2}$, (B.2) implies that the series $\sum_{n \geqq 0} R^{(n)}(z)(\omega ; \mathbf{x}, \mathbf{y})$ converges uniformly to a function bounded by $C \exp (-m|\mathbf{x}-\mathbf{y}|)$. By definition the sum is nothing but the integral kernel of $R_{\varepsilon}(\omega ; z)$. Now $R^{(n)}(z)(\omega ; \mathbf{x}, \mathbf{y})$ is continuous in $(\omega ; \mathbf{x}, \mathbf{y})$. For it can be written as:

$$
\begin{aligned}
R^{(n)}(z)(\omega ; \mathbf{x}, \mathbf{y})= & \int d^{2} \mathbf{x}_{1} \cdots d^{2} \mathbf{x}_{n} G_{\boldsymbol{B}}^{\tilde{(}}\left(\mathbf{x}_{1}, \mathbf{x}\right)^{*} G_{B}\left(\mathbf{x}_{1}, \mathbf{x}_{2}\right) \\
& \cdots G_{B}\left(\mathbf{x}_{n-1}, \mathbf{x}_{n}\right) G_{B}^{\tilde{B}}\left(\mathbf{x}_{n}, \mathbf{y}\right) V\left(\mathbf{x}_{1}\right) \cdots V\left(\mathbf{x}_{n}\right),
\end{aligned}
$$

where $G_{\boldsymbol{B}}^{\tilde{B}}(\mathbf{x}, \mathbf{y})$ is the integral kernel of $\left(H_{B}-z\right)^{-1}\left(\mathbf{1}+\varepsilon H_{B}\right)^{-1}$. Using the estimate (B.3) and the dominated convergence theorem, we get continuity with respect to $(\mathbf{x}, \mathbf{y})$ uniformly with respect to $\omega \in \Omega$. On the other hand, for a fixed $(\mathbf{x}, \mathbf{y})$, the integrand is the product of $V\left(\mathbf{x}_{1}\right) \cdots V\left(\mathbf{x}_{n}\right)$ by a fixed $L^{1}$ function. Thus it is continuous with respect to $V$ with the weak topology of $L^{1}$.

2) Let us now suppose $z \in \bigcap_{\omega \in \Omega} \rho\left(H_{\omega}\right)$. Then

$$
R_{\varepsilon}(z)(\omega ; \mathbf{x}, \mathbf{y})=\left\langle\left(\mathbf{1}+\varepsilon H_{B}\right)^{-1} \delta_{\mathbf{x}} \mid\left(H_{\omega}-z\right)^{-1}\left(\mathbf{1}+\varepsilon H_{B}\right)^{-1} \delta_{\mathbf{y}}\right\rangle .
$$

Since $\sup \left\|\left(1+\varepsilon H_{B}\right)^{-1} \delta_{\mathbf{x}}\right\|_{L^{2}}<\infty$ for $\varepsilon>0, R_{\varepsilon}(z)$ defines a complex analytic function in $\bigcap_{\omega \in \Omega} \rho\left(H_{\omega}\right)$ with values in $L^{\infty}\left(\Omega \times \mathbf{R}^{2} \times \mathbf{R}^{2}\right)$. Moreover, for $\operatorname{Re}(z)<-$ $m^{2}-\|V\|$, we have shown that $R_{\varepsilon}(z)$ takes on values in the closed subspace $\mathscr{C}_{0}\left(\Omega \times \mathbf{R}^{2} \times \mathbf{R}^{2}\right)$ of continuous function vanishing as $|\mathbf{x}-\mathbf{y}|$ tends to $\infty$. By the Lemma B.4, we conclude that indeed $R_{\varepsilon}(z) \in \mathscr{C}_{0}\left(\Omega \times \mathbf{R}^{2} \times \mathbf{R}^{2}\right)$ for $z \in \bigcap_{\omega \in \Omega} \rho\left(H_{\omega}\right)$.

Proof of Proposition B.2. From Lemma B.5 and the proof of Proposition B.1, $R_{\varepsilon}(z)$ belongs to $\mathcal{O}$ for $\operatorname{Re}(z)<-m^{2}-\|V\|$. Since $\mathcal{O}$ is a closed subspace of the Banach space $B\left(L^{2}\left(\mathbf{R}^{2}\right)\right)$, Lemma B.4, shows that $R_{\varepsilon}(z)$ belongs to $\mathcal{O}$ for $z \in \bigcap_{\omega \in \Omega} \rho\left(H_{\omega}\right)$. On 
the other hand,

$$
\begin{aligned}
\left\|R_{\varepsilon}(z)-R(z)\right\| & \leqq 2\left\|\left(H_{\omega}-z\right)^{-1} \varepsilon H_{B}\left(1+\varepsilon H_{B}\right)^{-1}\right\| \\
& \leqq 2 \varepsilon\left\|\left(H_{\omega}-z\right)^{-1}\left(H_{\omega}-V_{\omega}\right)\left(1+\varepsilon H_{B}\right)^{-1}\right\| .
\end{aligned}
$$

Thus the left-hand side converges to zero as $\varepsilon \rightarrow 0$ concluding the proof of Proposition B.2.

Lemma B.6. There is $\tau>0$ and for any $\gamma>0$, there is $C_{\gamma}>0$ such that uniformly in $\omega$ and $\varepsilon>0$ :

$$
\left|k_{\varepsilon}(\omega ; \mathbf{x}, \mathbf{y})\right| \leqq C_{\gamma} \exp (-\tau|\mathbf{x}-\mathbf{y}|) \quad \text { if } \quad|\mathbf{x}-\mathbf{y}| \geqq \gamma . \diamond
$$

Proof. Let $L_{v}^{(0)}$ be given by (A.4) with $V=0$. Noting that

$$
e^{i v \cdot \mathbf{x}} R_{\varepsilon}(z) e^{-i v \cdot \mathbf{x}}=\left(1+\varepsilon L_{v}^{(0)}\right)^{-1}\left(L_{v}-z\right)^{-1}\left(1+\varepsilon L_{v}^{(0)}\right)^{-1},
$$

we can apply the argument of Lemmas A.5-A.7 for $\boldsymbol{R}_{\varepsilon}$ analogously, to obtain (B.7) independently of $\varepsilon$ and $\omega$. Analogously to Lemma A.8, we obtain:

Lemma B.7. For $z \in \rho(H),\left[\mathbf{X}, R_{\varepsilon}(z)\right]$ has a continuous integral kernel given by $(\mathbf{x}-\mathbf{y}) k_{\varepsilon}(\omega ; \mathbf{x}, \mathbf{y})$ which satisfies for some $\tau>0$ and every $\mathbf{x}, \mathbf{y}$ :

$$
\left|(\mathbf{x}-\mathbf{y}) k_{\varepsilon}(\omega ; \mathbf{x}, \mathbf{y})\right| \leqq C \exp (-\tau|\mathbf{x}-\mathbf{y}|),
$$

where $C$ is independent of $\varepsilon$ and $\omega$.

\section{Appendix C. Proof of Connes Formula}

Here we give a proof of Theorem 5.4. At first we note:

Lemma C.1. The map $\left(A_{0}, A_{1}, A_{2}\right) \rightarrow \tau_{2}\left(\pi_{\omega}\left(A_{0}\right), \pi_{\omega}\left(A_{1}\right), \pi_{\omega}\left(A_{2}\right)\right)$ is continuous from $X^{x 3}$ into $L^{1}(\Omega)$. $\diamond$

Proof. By Lemma A.4, the map $A \rightarrow\left[F, \pi_{\omega}(A)\right]$ is bounded from $X$ to $L^{3}\left(\Omega ; \mathscr{L}^{3}(\mathscr{H})\right)$. The lemma follows from the definition of the 2-cocycle $\tau_{2}$. $\diamond$

Since $\mathcal{O}_{0}$ is dense in $\mathcal{O}, X$ and $\mathscr{H}^{1}$, it suffices to show the formula for $A_{i}$ in $\mathcal{O}_{0}$ by virtue of Lemmas 3.2 and C.1.

Lemma C.2. The Connes formula (5.6) holds for $A_{i}$ in $\mathcal{O}_{0}, i=0,1,2$.

Proof. By definition,

$$
\begin{aligned}
& \tau_{2}\left(\pi_{\omega}\left(A_{0}\right), \pi_{\omega}\left(A_{1}\right), \pi_{\omega}\left(A_{2}\right)\right) \\
&=-1 / 2 \int_{\mathbf{R}^{6}} d^{2} \mathbf{x}_{0} d^{2} \mathbf{x}_{1} d^{2} \mathbf{x}_{2}\left\{D ( \mathbf { x } _ { 0 } ) ^ { * } ( D ( \mathbf { x } _ { 0 } ) - D ( \mathbf { x } _ { 1 } ) ) \left(D\left(\mathbf{x}_{1}\right)^{*}\right.\right. \\
&\left.\left.-D\left(\mathbf{x}_{2}\right)^{*}\right)\left(D\left(\mathbf{x}_{2}\right)-D\left(\mathbf{x}_{0}\right)\right)-\text { c.c. }\right\} \\
& \times \cdots \times A_{0}\left(T^{-\mathbf{x}_{0}} \omega, \mathbf{x}_{1}-\mathbf{x}_{0}\right) A_{1}\left(T^{-\mathbf{x}_{1}} \omega, \mathbf{x}_{2}-\mathbf{x}_{1}\right) A_{2}\left(T^{-\mathbf{x}_{2}} \omega, \mathbf{x}_{0}-\mathbf{x}_{2}\right) \\
& \cdot e^{i B\left(\mathbf{x}_{0} \wedge \mathbf{x}_{1}+\mathbf{x}_{1} \wedge \mathbf{x}_{2}+\mathbf{x}_{2} \wedge \mathbf{x}_{0}\right) / 2},
\end{aligned}
$$

where $D(\mathbf{x})=\left(\mathbf{x}_{1}+\mathbf{i x} \mathbf{x}_{2}\right) /|\mathbf{x}|, \quad D(\mathbf{x})^{*}=\left(x_{1}-\mathbf{i} x_{2}\right) /|\mathbf{x}|$, and c.c. means "complex 
conjugate." By a direct computation, we have

$$
\begin{aligned}
& \left\{D\left(\mathbf{x}_{0}\right)^{*}\left(D\left(\mathbf{x}_{0}\right)-D\left(\mathbf{x}_{1}\right)\right)\left(D\left(\mathbf{x}_{1}\right)^{*}-D\left(\mathbf{x}_{2}\right)^{*}\right)\left(D\left(\mathbf{x}_{2}\right)-D\left(\mathbf{x}_{0}\right)\right)-\text { c.c. }\right\} \\
& \quad=-2 \beta\left(\mathbf{x}_{0}-\mathbf{x}_{1}, \mathbf{x}_{0}\right) \beta\left(\mathbf{x}_{1}-\mathbf{x}_{2}, \mathbf{x}_{1}\right) \beta\left(\mathbf{x}_{2}-\mathbf{x}_{0}, \mathbf{x}_{2}\right),
\end{aligned}
$$

with $\beta(\mathbf{x}, \mathbf{y})=1-D(\mathbf{x})^{-1} D(\mathbf{x}-\mathbf{y})^{-1}$. We perform the following change of variable and then integrate over $\omega$ :

$$
\begin{aligned}
& \mathbf{s}=\mathbf{x}_{0}, \mathbf{s}_{0}=\mathbf{x}_{0}-\mathbf{x}_{1}, \mathbf{s}_{1}=\mathbf{x}_{1}-\mathbf{x}_{2}, \mathbf{s}_{2}=\mathbf{x}_{2}-\mathbf{x}_{0}, \omega^{\prime}=T^{-\mathbf{s}} \omega ; \\
& \int_{\omega \in \Omega} \mathbf{P}(d \omega) \tau_{2}\left(\pi_{\omega}\left(A_{0}\right), \pi_{\omega}\left(A_{1}\right), \pi_{\omega}\left(A_{2}\right)\right) \\
& =\int_{\omega \in \Omega} \mathbf{P}(d \omega) \int_{\mathbf{R}^{6}} d^{2} \mathbf{s} d^{2} \mathbf{s}_{0} d^{2} \mathbf{s}_{1} \beta\left(\mathbf{s}_{0}, \mathbf{s}\right) \beta\left(\mathbf{s}_{1}, \mathbf{s}-\mathbf{s}_{1}\right) \beta\left(\mathbf{s}_{2}, \mathbf{s}-\mathbf{s}_{1}-\mathbf{s}_{2}\right) \\
& \times \cdots \times A_{0}\left(\omega^{\prime},-\mathbf{s}_{0}\right) A_{1}\left(T^{\mathbf{s}_{0}} \omega^{\prime},-\mathbf{s}_{1}\right) A_{2}\left(T^{\mathbf{s}_{0}+\mathbf{s}_{1}} \omega^{\prime},-\mathbf{s}_{2}\right) e^{i \mathbf{s}_{0} \wedge \mathbf{s}_{1} / 2} .
\end{aligned}
$$

Here we integrate over the subspace $\left\{\mathbf{s}_{0}+\mathbf{s}_{1}+\mathbf{s}_{2}=0\right\}$. These integrals are absolutely convergent because they are compactly supported with respect to the variables $\mathbf{s}_{i}$, and decay like $\langle\mathbf{s}\rangle^{-\mathbf{3}}$ in s. Using [see [27], Part I, Lemma 9.2]:

$$
\int_{\mathbf{R}^{2}} d^{2} \mathbf{s} \beta\left(\mathbf{s}_{0}, \mathbf{s}\right) \beta\left(\mathbf{s}_{1}, \mathbf{s}-\mathbf{s}_{1}\right) \beta\left(\mathbf{s}_{2}, \mathbf{s}-\mathbf{s}_{1}-\mathbf{s}_{2}\right)=2 \mathbf{i} \pi \mathbf{s}_{0} \wedge \mathbf{s}_{1},
$$

we obtain:

$$
\begin{aligned}
\int_{\omega \in \Omega} \mathbf{P}(d \omega) \tau_{2}\left(\pi_{\omega}\left(A_{0}\right), \pi_{\omega}\left(A_{1}\right), \pi_{\omega}\left(A_{2}\right)\right) \\
=\int \mathbf{P}(d \omega) \int_{\mathbf{R}^{4}} d^{2} \mathbf{s}_{0} d^{2} \mathbf{s}_{1} A_{0}\left(\omega^{\prime},-\mathbf{s}_{0}\right) A_{1}\left(T^{\mathbf{s}_{0}} \omega^{\prime},-\mathbf{s}_{1}\right) \\
\cdot A_{2}\left(T^{\mathbf{s}_{0}+\mathbf{s}_{1}} \omega^{\prime}, \mathbf{s}_{0}+\mathbf{s}_{1}\right) 2 \mathbf{i} \pi \mathbf{s}_{0} \wedge \mathbf{s}_{1} e^{i B \mathbf{s}_{0} \wedge \mathbf{s}_{1} / 2} \\
=1 / 2 \mathbf{i} \pi \tau\left(A_{0}\left\{\partial_{1} A_{1} \partial_{2} A_{2}-\partial_{2} A_{1} \partial_{1} A_{2}\right\}\right)=\tau_{2}\left(A_{0}, A_{1}, A_{2}\right) .
\end{aligned}
$$

\section{Appendix D. Equivalence of Projection in p-Summable Subalgebras}

Here we prove a criterion for equivalence of projections, which is well-known for $C^{*}$-Algebras, but we need it in the $p$-summable subalgebra $\mathscr{A}^{(p)}$.

Lemma D.1. Let $\mathscr{A}$ be a $C^{*}$-Algebra with a $\mathbf{Z}_{2}$-graded representation, and let $\mathscr{A}^{(p)}$ be the subalgebra of p-summable elements of $\mathscr{A}$. Suppose that $e$ and $f$ are two projection in $\mathscr{A}^{(p)}$ such that $\|e-f\|<1$, then $e$ and $f$ are equivalent in $\mathscr{A}^{(p)}$.

Proof. We let $\left\{c_{n}\right\}$ be the coefficients of the Taylor expansion on $(1-X)^{-1 / 2}=$ $\sum_{n \geqq 0} c_{n} X^{n}$, and define $(e f e)^{-1 / 2}:=e+\sum_{n \geqq 1} c_{n}(e-e f e)^{n}$. If we set $u=(e f e)^{-1 / 2} e f$, then $u$ satisfies $u^{*} u=f$ and $u u^{*}=e$. It remains only to show that $u \in \mathscr{A}^{(p)}$. By an easy computation,

$$
\begin{aligned}
{[F, u]=} & {[F, e] e f+\sum_{n \geqq 1} \sum_{k=1}^{n} c_{n}(e-e f)^{k-1}[F, e-e f](e-e f)^{n-k} e f } \\
& +\left(e+\sum_{n \geqq 1} c_{n}(e-e f e)^{n}\right)[F, e f] .
\end{aligned}
$$


Noting that $e-e f$ and $e f$ are $p$-summable, we get:

$$
\begin{aligned}
\|[F, u]\|_{p} \leqq & \|[F, e]\|_{p}+\|[F, e-e f]\|_{p} \sum_{n \geqslant 0} n\left|c_{n}\right|\|e-f\|^{n} \\
& +\sum_{n \geqslant 0}\left|c_{n}\right|\|e-f\|^{n}\|[F, e f]\|_{p},
\end{aligned}
$$

where $\|\cdot\|_{p}$ denotes the $p^{\text {th }}$ trace norm on $\mathscr{H}$. The right-hand side of (D.2) converges because $\|e-f\|$ is smaller than 1 and the convergence radius of $\sum_{n \geqslant 0} n\left|c_{n}\right| X^{n}$ is
one. Hence $[F, u] \in \mathscr{L}^{(p)}$.

Acknowledgements. J. B. wants to thank F. Axel for explaining her results prior to publication. S. N. wants to thank the Centre de Physique Théorique and the Université de Provence at Marseille for its hospitality and for providing financial support and facilities while this work was done.

\section{References}

1. Landau, L.: Diamagnetismus der Metalle. Z. Phys. 64, 629-637 (1930)

2. Thouless, D., Kohmoto, M., Nightingale, M., M. den Nijs: Quantized Hall conductance in two dimensional periodic potential, Phys. Rev. Lett. 49, 405 (1982)

3. Avron, J., Seiler, R.: Quantization of the Hall conductance for general multiparticle Schrœdinger hamiltonians. Phys. Rev. Lett. 54, 259-262 (1985)

4. Kunz, H.: The Quantum Hall effect for electrons in a random potential. Commun. Math. Phys. 112, 121-145 (1987)

5. Bellissard, J.: K-Theory of $\mathrm{C}^{*}$-algebras in solid state physics. Statistical Mechanics and Field theory, Mathematical aspects. Dorlas, T. C., Hugenholtz, M. N., Winnink, M. (eds) pp. 99-156. Lecture Note in Physics, vol. 257. Berlin, Heidelberg, New York: Springer 1986

6. Bellissard, J.: Ordinary quantum Hall effect and non-commutative cohomology. In: Localization in disordered systems. Weller, W., Ziesche, P. (eds). Leipzig: Teubner 1987

7. Bellissard, J.: $C^{*}$-Algebras in Solid State Physics: 2D Electrons in a uniform magnetic field. In: Operator algebras and applications, vol. II. Evans, E. V., Takesaki, M. (eds). Cambridge: Cambridge University Press 1988

8. Xia, J.: Geometric invariants of the quantum Hall effect. Commun. Math. Phys. 119, 29-50(1988)

9. Tesanovic, Z., Axel, F., Halperin, B.: "Hall crystal" versus Wigner crystal. Phys. Rev. B39, 8525-8551 (1989)

10. Harper, P. G.: Single band motion of conduction electrons in a uniform magnetic field. Proc. Phys. Soc. Lond. A68, 874 (1955)

11. Nenciu, G.: Bloch electrons in a magnetic field: Rigorous justification of the Peierls-Onsager effective hamiltonian. Preprint Buchuresti, (April 1988)

12. Helffer, B., Sjöstrand, J.: Analyse semi classique pour l'équation de Harper, (avec application à l'étude de l'équation de Schrödinger en champ magnétique), I, II, III. Preprint University Orsay, (1988), to appear in Bull. Soc. Math. France.

13. Hofstadter, D. R.: Energy levels and wave functions of Bloch electrons in a rational or irrational magnetic field. Phys. Rev. B14, 2239 (1976)

14. Wilkinson, M.: Critical properties of electrons eigenstates in Incommensurate systems. Proc. Roy. Soc. Lond. A391, 305-350 (1984)

15. Bellissard, J., Simon, B.: Cantor spectrum for the Almost Mathieu equation, J. Func. Anal. 48, 408-419 (1982)

16. Sinai, Ya. G.: Anderson localization for a one-dimensional difference Schrödinger operator with quasiperiodic potential. J. Stat. Phys. 46, 861-909 (1987)

17. Van Mouche, P.: The coexistence problem for the discrete Mathieu operator. Commun. Math. Phys. 122, 23-33 (1989)

18. Choi, Elliott, G., Yui, N.: Gauss polynomials and the rotation algebra. To appear in Inv. Math. (1990) 
19. Briet, P., Combes, J. M., Duclos, P.: On the location of Resonances for Schrödinger operators in the semiclassical limit II. Comm. P.D.E. 12, 201-222 (1987)

20. Helffer, B., Sjöstrand, J.: (i)-Multiple Wells in the semiclassical limit I, Comm. P.D.E. 9, 337-408 (1984) (ii)-Puits Multiples en Analyse Semiclassique II. Ann. I.H.P. 42, 127-212 (1985)

21. Reed, M., Simon, B.: Methods of modern mathematical physics, vol. I-IV. London, New York: Academic Press 1978

22. Zak, J.: Magnetic translation group. Phys. Rev. A134, 1602-1607 (1964); Magnetic translation group II: irreducible representations. Phys. Rev. A134, 1607-1611 (1964)

23. Kubo, K.: Statistical mechanics. Amsterdam: North Holland 1967

24. Simon, B.: Semiclassical analysis of low-lying eigenvalues, I: Non-degenerate minima. Ann. Inst. H. Poincaré 38, 295-307 (1983)

25. Halmos, P. R.: Lectures on ergodic theory. New York: Chelsea 1956

26. Pedersen, G.: $C^{*}$-Algebras and their automorphism groups. London, New York: Academic Press 1979

27. Connes, A.: Non-commutative differential geometry. Pub. IHES 62, 43-144 (1986)

28. Kunz, H., Souillard, B.: Sur le spectre des opérateurs aux différences finies aléatoires. Commun. Math. Phys. 78, 201-246 (1981)

29. Atiyah, M.: K-Theory. New York, Amsterdam: Benjamin 1967

30. Briet, P., Combes, J. M., Duclos, P.: Spectral stability under tunneling. Commun. Math. Phys. 126, 133-156 (1989); Spectral stability under tunneling for Schrödinger operators. Proc. Conf. "Partial Differential Equations” Holzhau, DDR, 1988, Schultze \& Treibel Eds. Leipzig: Teubner Text 1989

31. Simon, B.: Trace ideals and their applications. London Math. Soc. Lecture Notes vol. 35, Cambridge: Cambridge University Press 1979

32. Simon, B.: Schrödinger semigroups. Bull A.M.S. 7, 447-526 (1982)

Communicated by B. Simon

Received July 25, 1989; in revised form December 8, 1989 
